\title{
How I curate: applying American Society of Hematology-Clinical Genome Resource Myeloid Malignancy Variant Curation Expert Panel rules for RUNX1 variant curation for germline predisposition to myeloid malignancies
}

Haematologica 2020

Volume 105(4):870-887

\section{Correspondence:}

DAVID WU

dwu2@uw.edu

LUCY A. GODLEY

Igodley@medicine.bsd.uchicago.edu

Received: July 16, 2019.

Accepted: October 21, 2019.

Pre-published: March 12, 2020

doi:10.3324/haematol.2018.214221

Check the online version for the most updated information on this article, online supplements, and information on authorship \& disclosures: www.haematologica.org/content/105/4/870

\section{(C)2020 Ferrata Storti Foundation}

Material published in Haematologica is covered by copyright. All rights are reserved to the Ferrata Storti Foundation. Use of published material is allowed under the following terms and conditions:

https://creativecommons.org/licenses/by-nc/4.0/legalcode. Copies of published material are allowed for personal or internal use. Sharing published material for non-commercial purposes is subject to the following conditions:

https://creativecommons.org/licenses/by-nc/4.0/legalcode, sect. 3. Reproducing and sharing published material for commercial purposes is not allowed without permission in writing from the publisher.

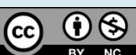

David Wu,,$^{1, *}$ Xi Luo, ${ }^{2 \star}$ Simone Feurstein, ${ }^{3}$ Chimene Kesserwan, ${ }^{4}$ Shruthi Mohan, ${ }^{5}$ Daniel E. Pineda-Alvarez, ${ }^{6}$ and Lucy A. Godley ${ }^{3,7}$ on behalf of the collaborative group of the American Society of Hematology - Clinical Genome Resource Myeloid Malignancy Variant Curation Expert Panel\#

${ }^{1}$ Department of Laboratory Medicine, University of Washington, Seattle, WA; ${ }^{2}$ Department of Pediatrics/Hematology-Oncology, Baylor College of Medicine, Houston, TX; ${ }^{3}$ Section of Hematology/Oncology, Department of Medicine, and The University of Chicago Comprehensive Cancer Center, Chicago, IL; ${ }^{4}$ Albert Einstein College of Medicine, Department of Pathology, New York, NY; ${ }^{5}$ Department of Genetics, University of North Carolina School of Medicine, Chapel Hill, NC; ${ }^{\circ}$ Invitae, San Francisco, CA and ${ }^{7}$ Department of Human Genetics, The University of Chicago, Chicago, IL, USA

${ }^{*} D W$ and $X L$ contributed equally to this work.

"Members of this Clinical Genome Resource Variant Curation Expert Panel are listed in the Acknowledgments.

\section{ABSTRACT}

The broad use of next-generation sequencing and microarray platforms in research and clinical laboratories has led to an increasing appreciation of the role of germline mutations in genes involved in hematopoiesis and lineage differentiation that contribute to myeloid neoplasms. Despite implementation of the American College of Medical Genetics and Genomics and Association for Molecular Pathology 2015 guidelines for sequence variant interpretation, the number of variants deposited in ClinVar, a genomic repository of genotype and phenotype data, and classified as having uncertain significance or being discordantly classified among clinical laboratories remains elevated and contributes to indeterminate or inconsistent patient care. In 2018, the American Society of Hematology and the Clinical Genome Resource co-sponsored the Myeloid Malignancy Variant Curation Expert Panel to develop rules for classifying gene variants associated with germline predisposition to myeloid neoplasia. Herein, we demonstrate application of our rules developed for the RUNX1 gene to variants in six examples to show how we would classify them within the proposed framework.

\section{Introduction}

Germline mutations in genes involved in hematopoiesis and lineage differentiation predispose patients to myeloid neoplasia with or without thrombocytopenia. The broad adoption of next-generation sequencing and microarrays in the clinical laboratory has expanded our knowledge of germline contribution to myeloid neoplasia. Drazer et al. reported that in six of 24 patients with myeloid neoplasia, presumed somatic variants in DDX41, GATA2 and TP53 were of germline origin. ${ }^{1}$ Similarly, Churpek et al. showed that $29 \%$ of acute myeloid leukemia (AML)/myelodysplastic syndrome (MDS) kindreds with a positive family history carried a variant in one of 12 genes associated with germline predisposition to hematopoietic malignancies, including FANCA, GATA2, RUNX1, and SBDS. ${ }^{2}$ To date, more than 65 genes have been associated with a predisposition to hematologic malignancies. ${ }^{3}$ Recognizing the contribution of germline variation toward myeloid neoplasia, the 'WHO classification of Tumors of Hematopoietic and 
Lymphoid Tissues' incorporated the classification of myeloid neoplasia with germline predisposition in their 2016 revised edition. ${ }^{4,5}$

In parallel, clinical laboratories are increasingly offering broad next-generation sequencing-based tests for patients with myeloid neoplasia for somatic testing, and will readily detect germline variants, if present in a patient. While there is increased clinical awareness of the potential for these germline variants to contribute to a patient's disease, there are often insufficient data in the literature to definitively classify whether a detected variant is contributing to the patient's phenotype. ${ }^{6,7}$ For example, familial platelet disorder with predisposition to AML (FPD/AML) is an autosomal dominant disorder in which germline mutations in $R U N X 1$ result in thrombocytopenia, platelet functional and/or ultrastructural defects, and/or susceptibility to hematologic malignancies commonly including MDS, AML, and other malignancies ${ }^{8-11}$ (Table 1). ClinVar (https://www.ncbi.nlm.nih.gov/clinvar) is a database repository of clinically actionable genomic variants $^{12,13}$ that currently lists 325 germline RUNX1 variants deposited by clinical laboratories. More than half of these variants are currently reported as being of uncertain significance.

Worldwide, most clinical laboratories follow the 2015 American College of Medical Genetics and Genomics (ACMG) and the Association for Molecular Pathology (AMP) guidelines for sequence variant interpretation. ${ }^{14}$ In this framework, germline variants are classified using a five-tier system: benign (BEN), likely benign (LBEN), variant of uncertain significance (VUS), likely pathogenic (LPATH) and pathogenic (PATH). During sequence variant interpretation, laboratories systematically review the supporting criteria of a genomic variant, such as: minor allele frequencies (MAF), computational predictions, functional experiments and segregation with disease in order to determine the five-tier classification. ${ }^{14-16}$

Although the ACMG/AMP guidelines provide a comprehensive framework for sequence variant interpretation, the high rate of VUS and curation discrepancies continue to be an impediment to accurate clinical annotation and interpretation of genomic variants. ${ }^{6,7}$ To encourage genomic and phenotypic data sharing, and engage experts in consensus-driven variant interpretation, the Clinical Genome Resource (ClinGen) convened Variant Curation Expert Panels (VCEP) to develop gene- and disease-specific modifications of the original guidelines and provide expert-reviewed variant classification for depositing into ClinVar (Online Supplementary Figure S1). ${ }^{17}$ In 2018, the American Society of Hematology (ASH) sponsored a ClinGen Myeloid Malignancy Variant Curation Expert
Panel (MM-VCEP), composed of 34 international members, who started working on gene- and disease-specific rules for RUNX1 as the first of several genes conferring predisposition to myeloid malignancies (Online Supplementary Figure S1A). After designing, modifying and testing the preliminary RUNX1 rules on 52 pilot variants, which improved classification in $33 \%$ VUS or variants with conflicting interpretations (CONF), MM-VCEPspecified ACMG/AMP rules were approved by the ClinGen oversight committee and efforts to curate variants to ClinVar using the Variant Curation Interface have commenced (Online Supplementary Figure S1B)..$^{18}$ This pilot effort resulted in one variant being upgraded to PATH, two variants being upgraded to LPATH, and three variants being downgraded to LBEN. ClinGen's website contains the MM-VCEP variant classification recommendations and any subsequent modifications to these codes over time (https://www.clinicalgenome.org/affiliation/10034/).

Herein, we demonstrate the application of RUNX1-specific rules (Table 2 ) to classify nine representative $R U N X 1$ variants in six examples (Table 3 ) while reviewing phenotypic criteria for FPD/AML and summarizing molecular and functional roles of RUNX1.

Example 1. Early nonsense variants, (p.Arg204Ter) (PATH with PVS1, PM2, PS4_supporting, and PP1)

A 50-year old female with new pancytopenia was referred to a hematology service. A bone marrow biopsy showed hypocellularity with severe trilineage dysplasia and $12 \%$ blasts, diagnostic of MDS with excess blasts (MDSEB-2). Further investigation showed pathogenic variants in RUNX1 (NM_001754:c.610C>T, (p.Arg204Ter)), BCOR and ASXL1 with a normal karyotype. The medical history was positive for thrombocytopenia (baseline 70 $120 \times 10^{9} / \mathrm{L}$ ) and a propensity to excessive bleeding after tooth extractions. The family history was positive for two sons with persistent thrombocytopenia (baseline 50$100 \times 10^{9} / \mathrm{L}$ ) not otherwise explained and a granddaughter with thrombocytopenia and MDS with monosomy 7 (Figure 1). During the initial assessment, an increase in lactate dehydrogenase and the peripheral blast count were noted. A second marrow biopsy confirmed transformation into AML with $40 \%$ blasts. The patient underwent induction chemotherapy without achieving remission and clofarabine bridging for unrelated stem cell transplantation. During conditioning, the patient developed sepsis with Gram-negative bacteria and died shortly afterwards. Since she had a remarkable personal and family history pointing towards a germline predisposition syndrome, a skin biopsy was performed at the time of the diagnosis of MDS, and DNA testing from cultured skin

Table 1. Clinical phenotypes of RUNX1 familial platelet disorder and hereditary malignancies.

\begin{tabular}{|c|c|c|}
\hline Clinical and laboratory features & Details & Life-time risk \\
\hline Hematologic malignancy & $\begin{array}{l}\text { Commonly AML or MDS; less frequently T-ALL; and rarely mixed MPN/MDS } \\
\text { such as CMML, as well as B-ALL, and hairy cell leukemia }\end{array}$ & $\sim 44 \%$ \\
\hline Thrombocytopenia & $\begin{array}{l}\text { Mild to moderate thrombocytopenia with normal platelet size, in the absence } \\
\text { of other causes }\end{array}$ & Most patients \\
\hline $\begin{array}{l}\text { Platelet functional and/or } \\
\text { ultrastructural defects }\end{array}$ & $\begin{array}{l}\text { Includes impaired platelet aggregation (particularly in response to collagen } \\
\text { and epinephrine) and platelet alpha or dense granule secretion defects }\end{array}$ & Not known \\
\hline
\end{tabular}

Adapted from Table 2 from Luo and Feurstein, et al. ${ }^{18}$ AML: acute myeloid leukemia; ALL: acute lymphoblastic leukemia; MPN: myeloproliferative neoplasms; MDS: myelodysplastic syndrome, CMML: chronic myelomonocytic leukemia. 

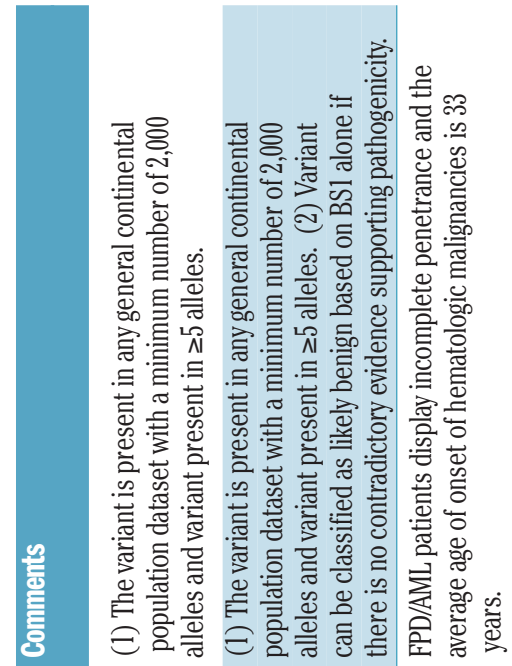


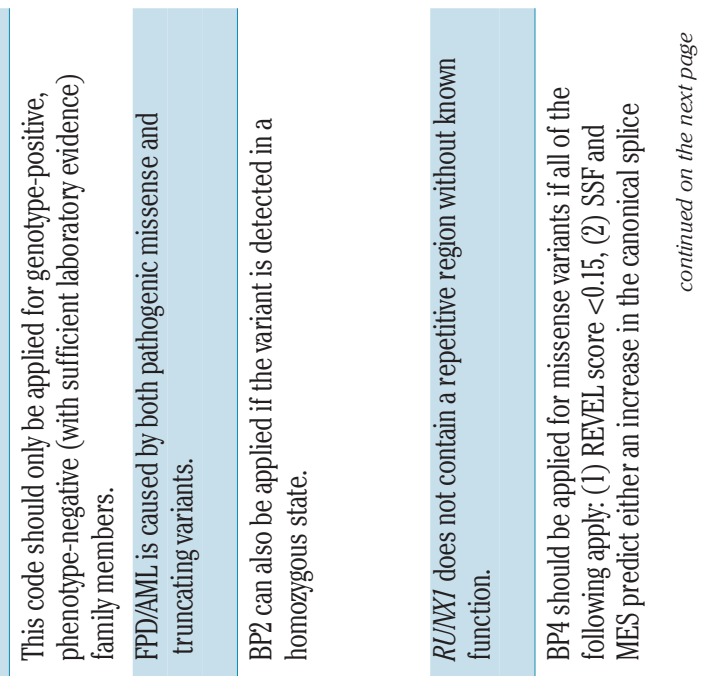

言 $\cong$
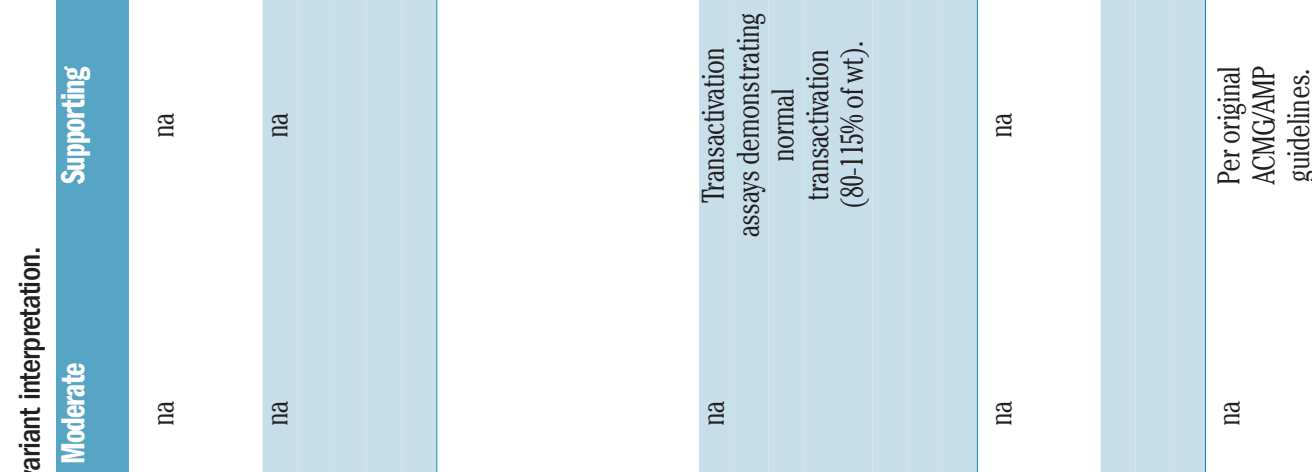

总商

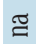

$\dddot{\Xi}$

$\cong$

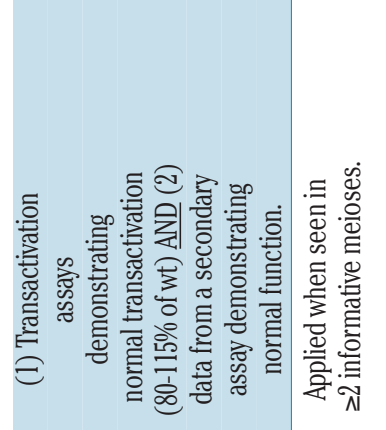

产

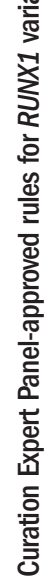

$\cong$

$\Xi$

๔

$\approx$

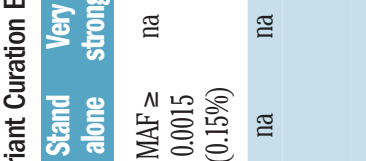

帝

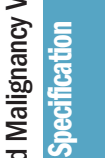

هُ

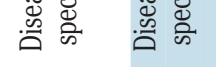

$\cong$

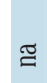

$\dddot{q}$

䢘高

$\Xi$

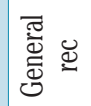

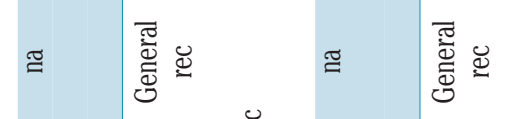

흘

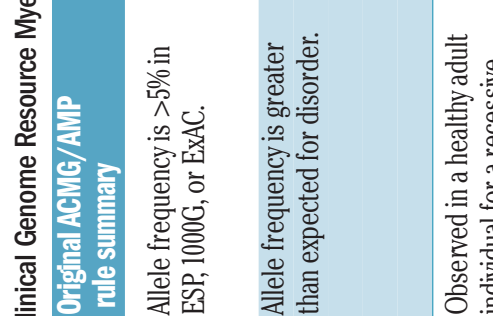

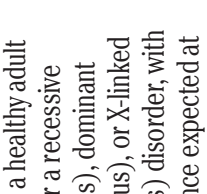

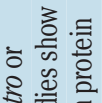

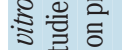

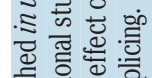

क्.

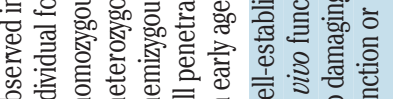

기이

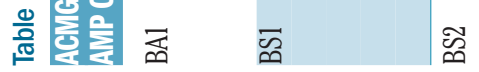

ஜ

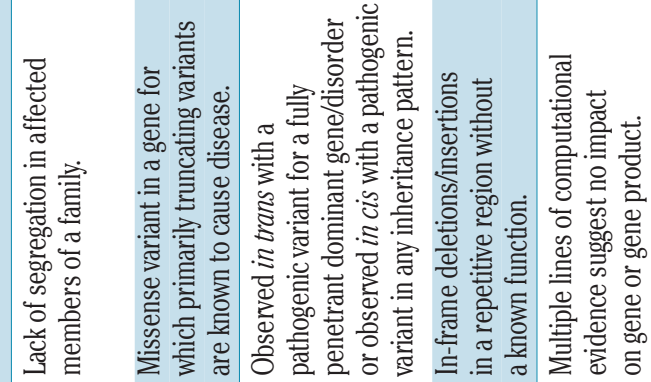

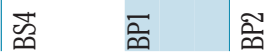

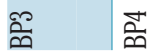




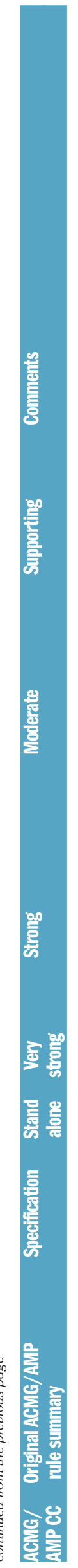

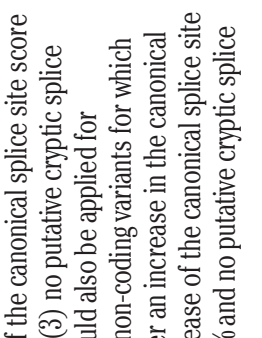

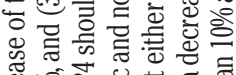

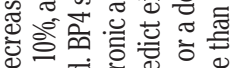

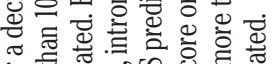

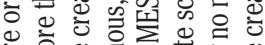

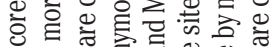

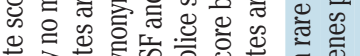
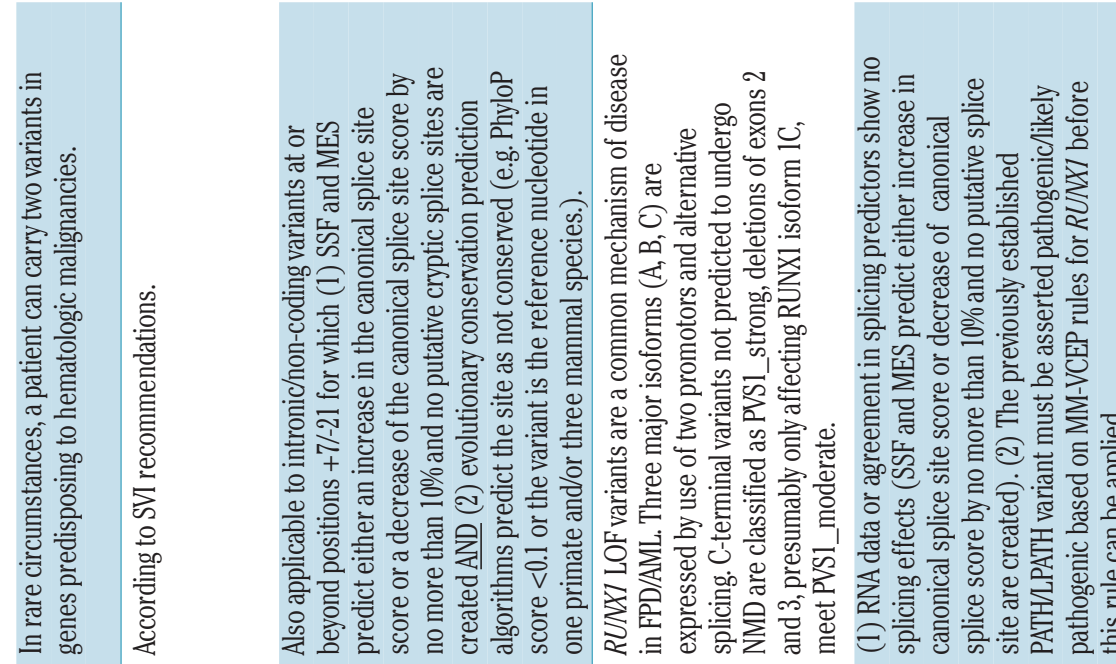

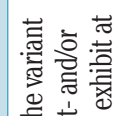
竞离兽兽 离 解 을.

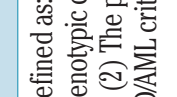

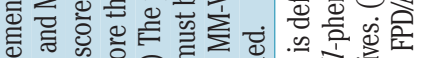

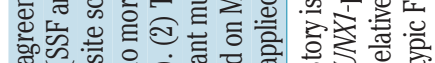
的。 하에

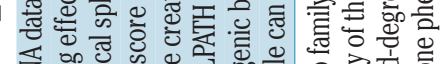

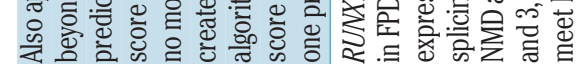

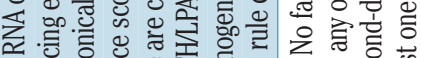

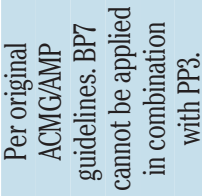

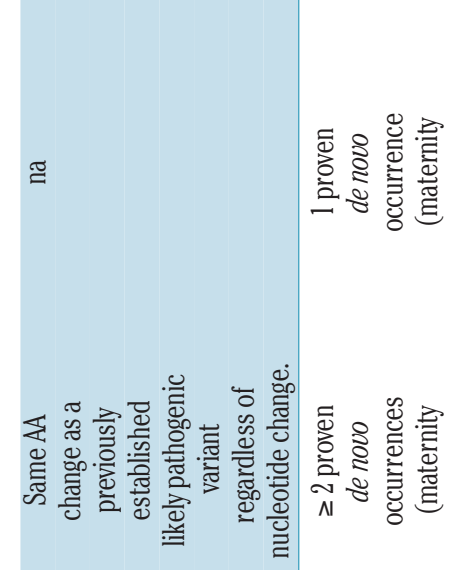

$\cong$

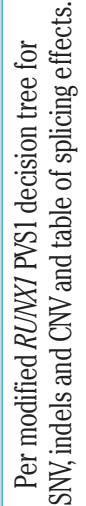

$\cong$

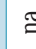
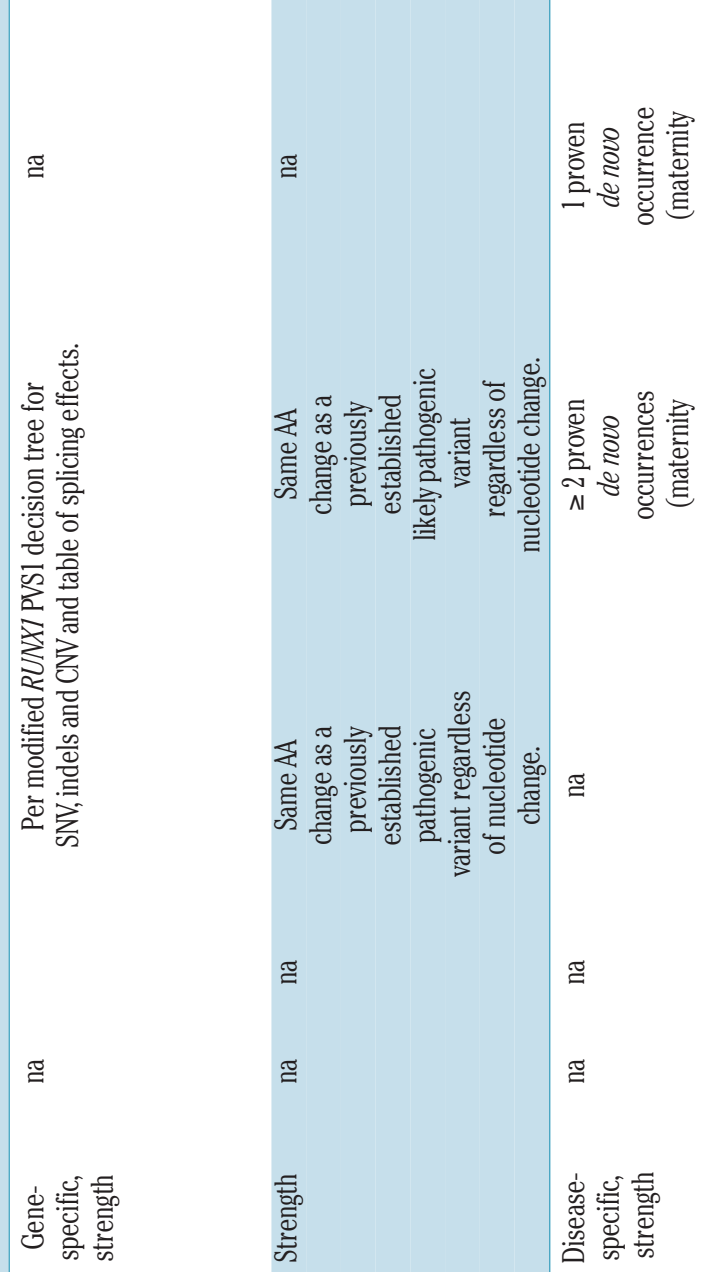

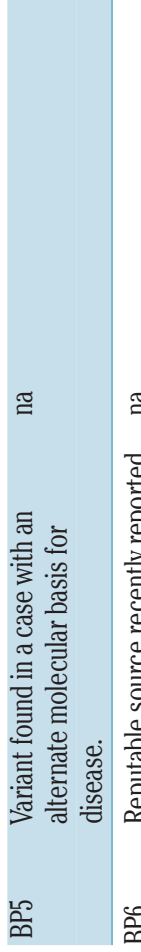
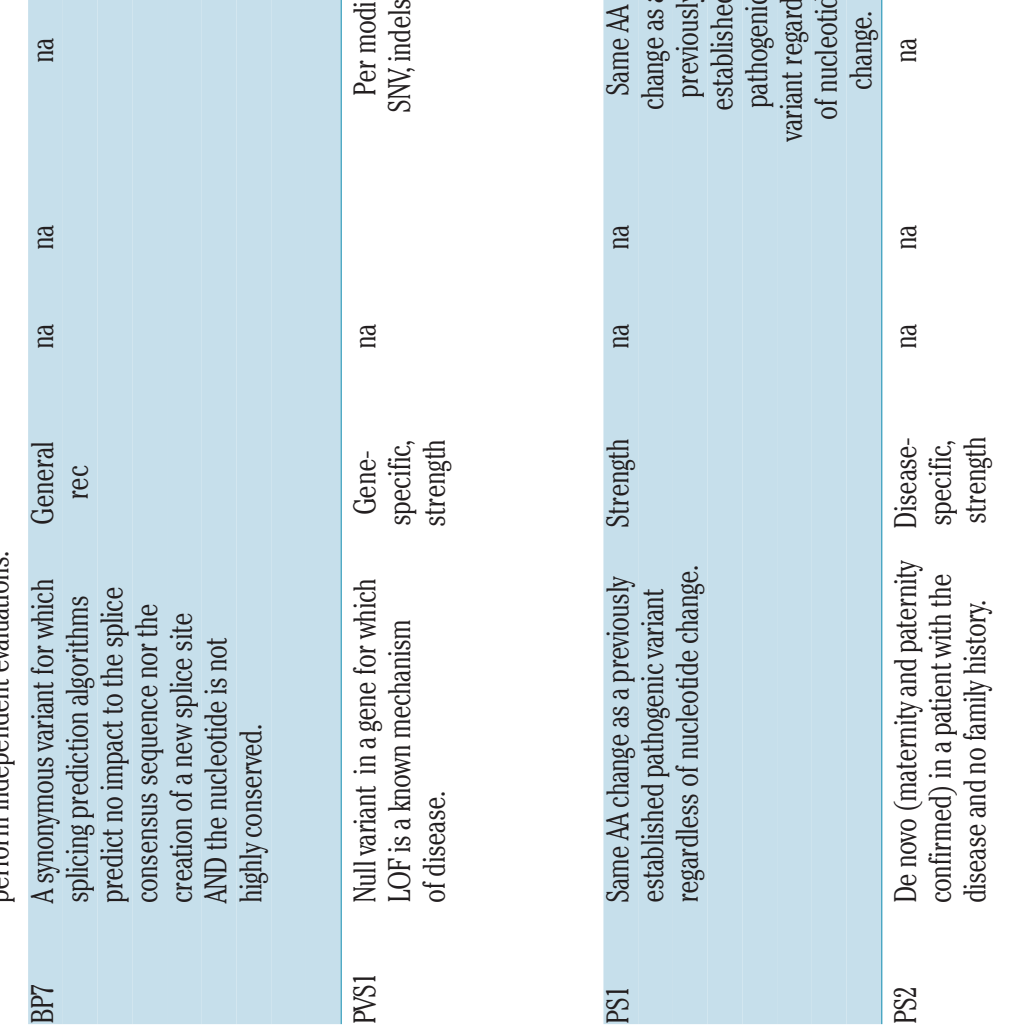

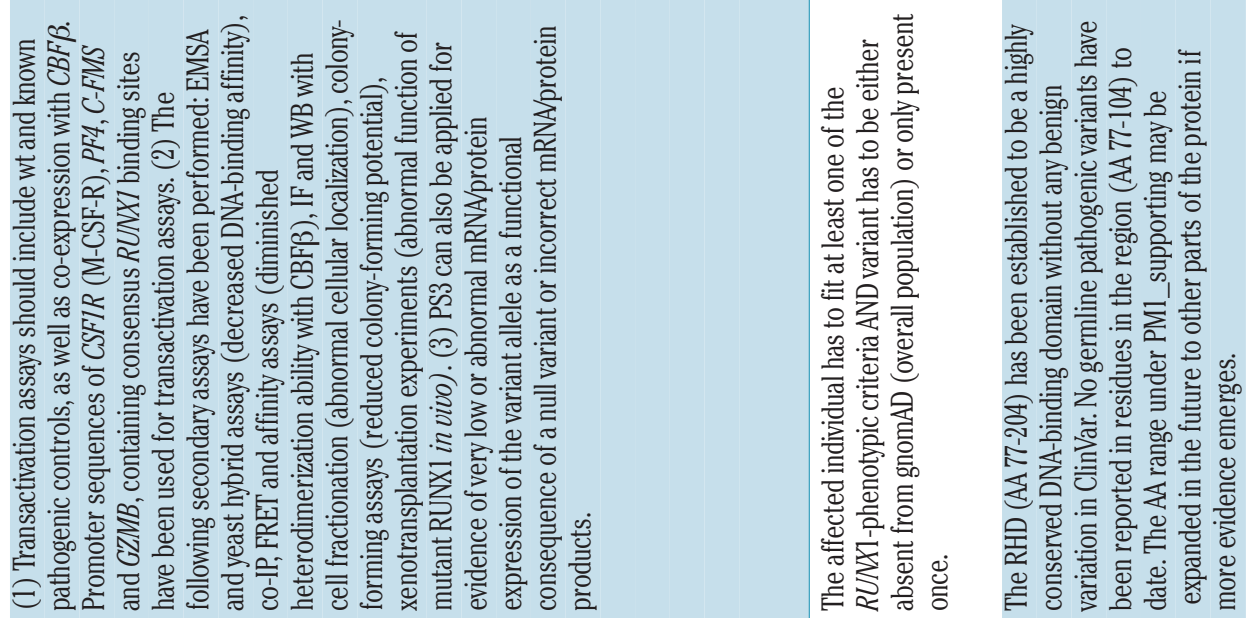

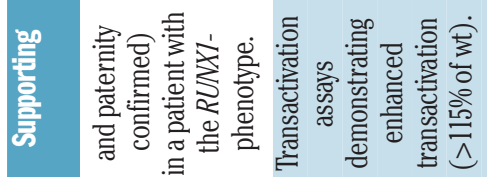

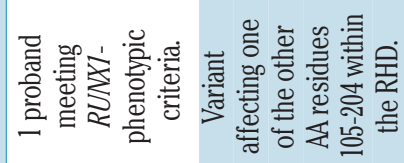

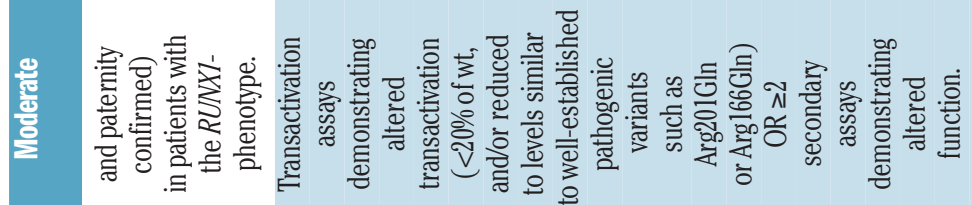

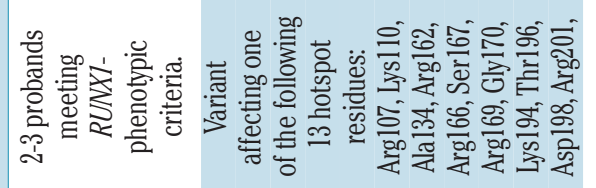

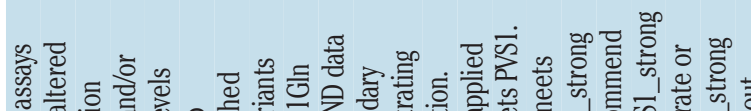

器

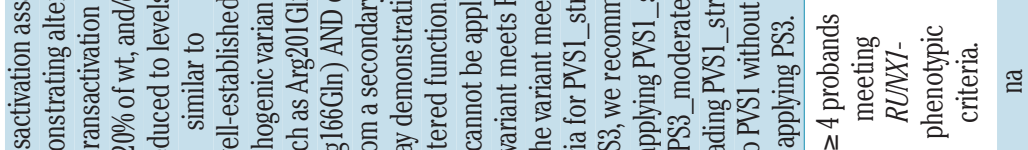

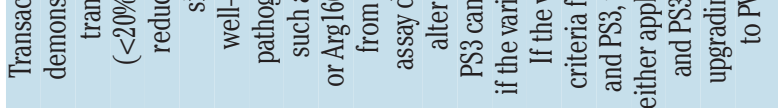

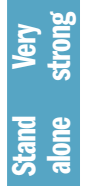

$\cong$

$\cong \quad \cong$

$\cong$

$\cong \quad \cong$

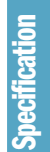

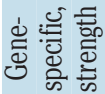

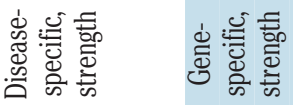

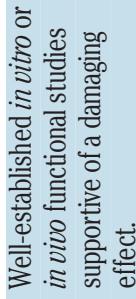

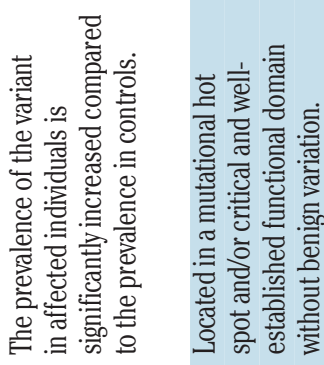

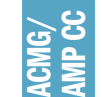

范

$\sum$ 


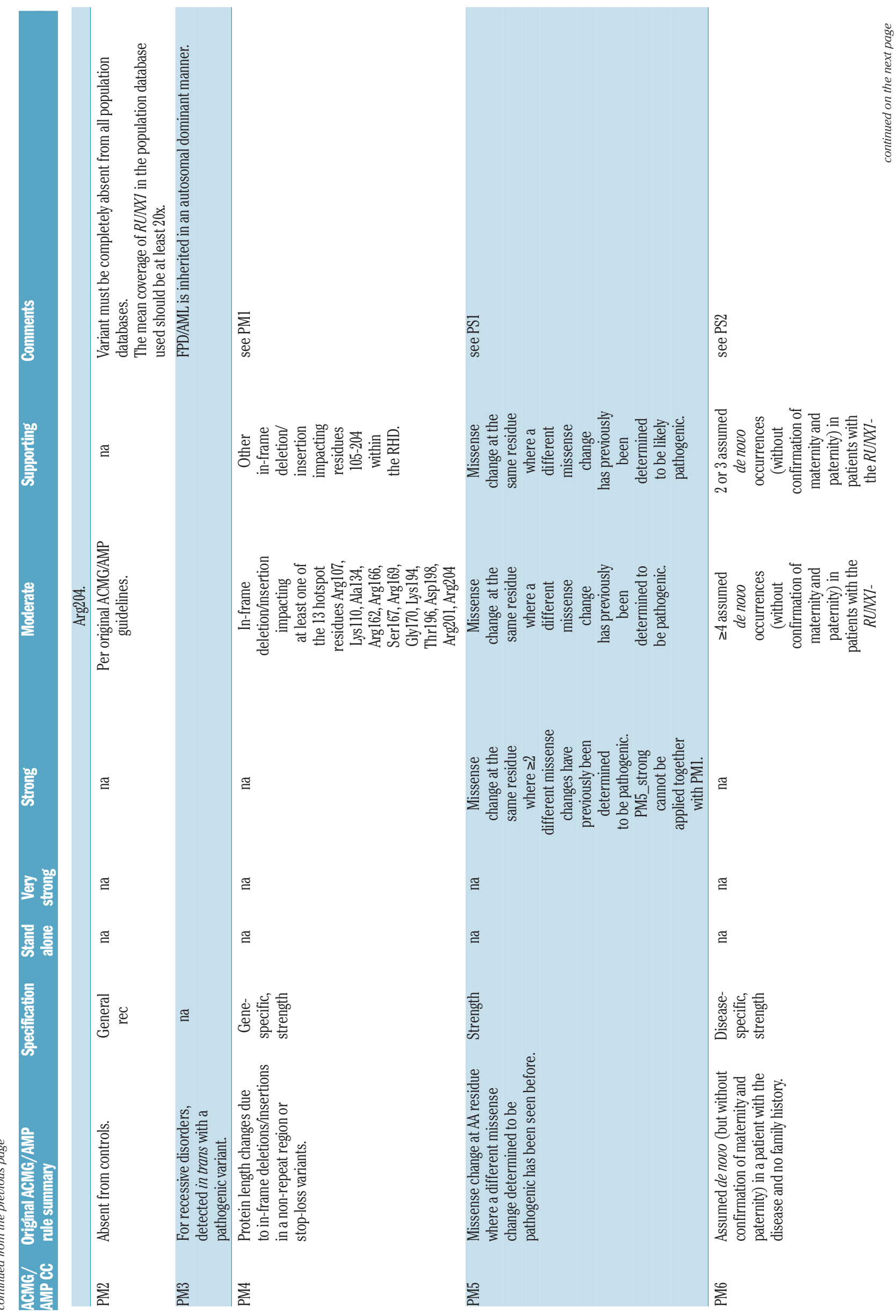



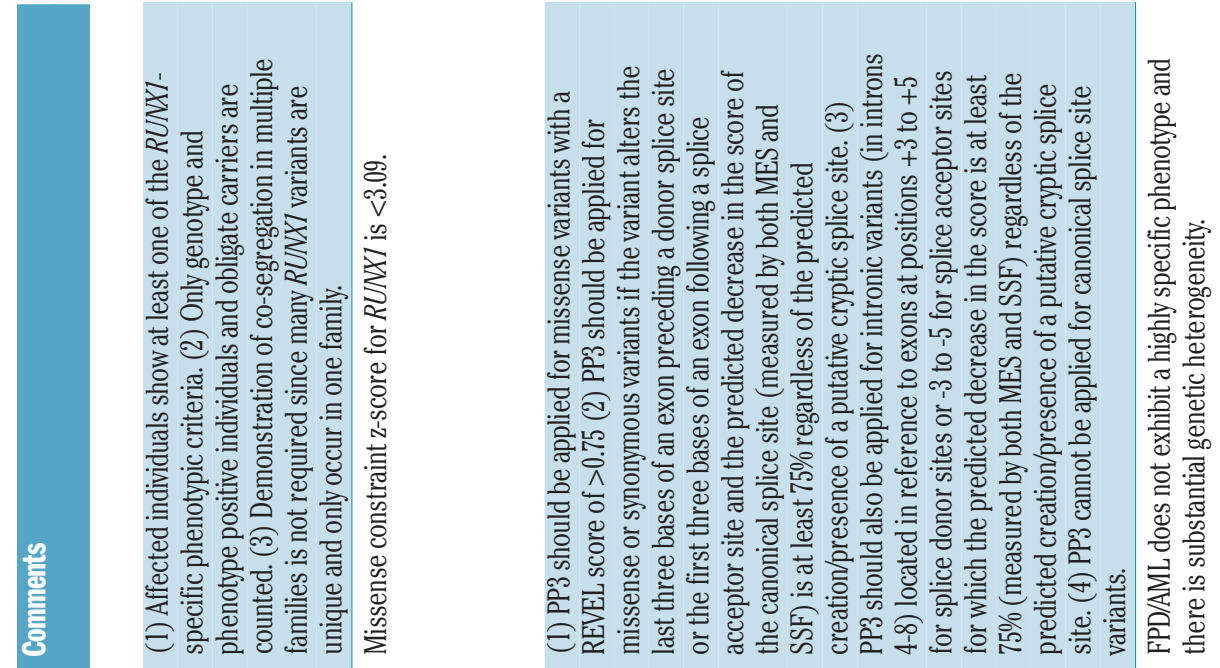

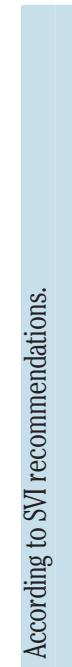

崖毫

䒠

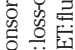
을

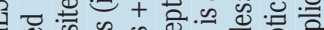

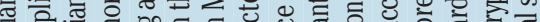
중 응.

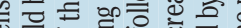
等

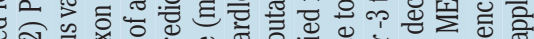

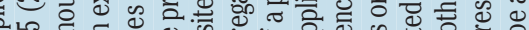

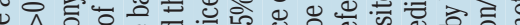
흐웡

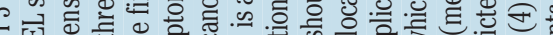

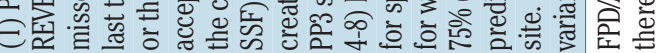

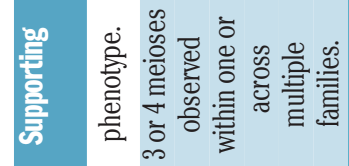

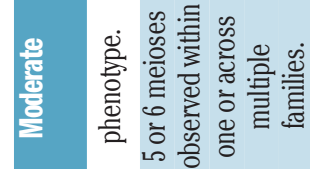

总

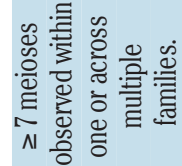

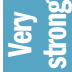

韾

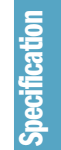

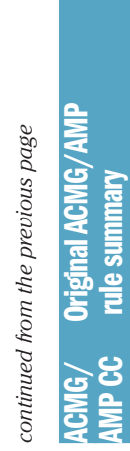

$\cong$

$\cong$

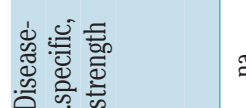

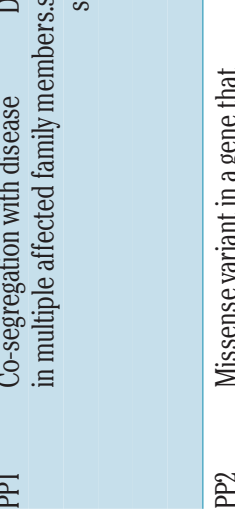

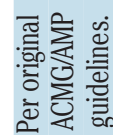

胥

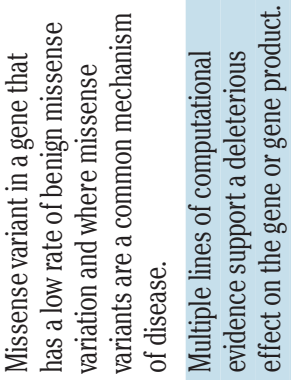

ฐ

๓̊ $\cong$

$\cong$

$\cong$

$\cong$

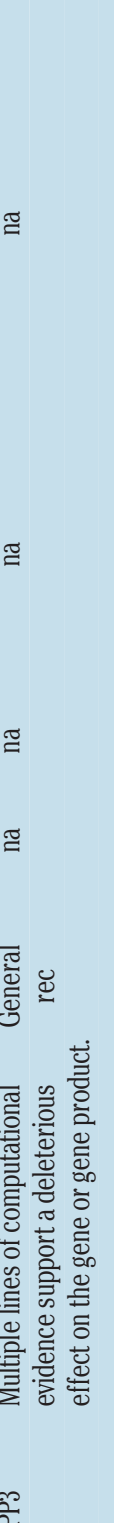

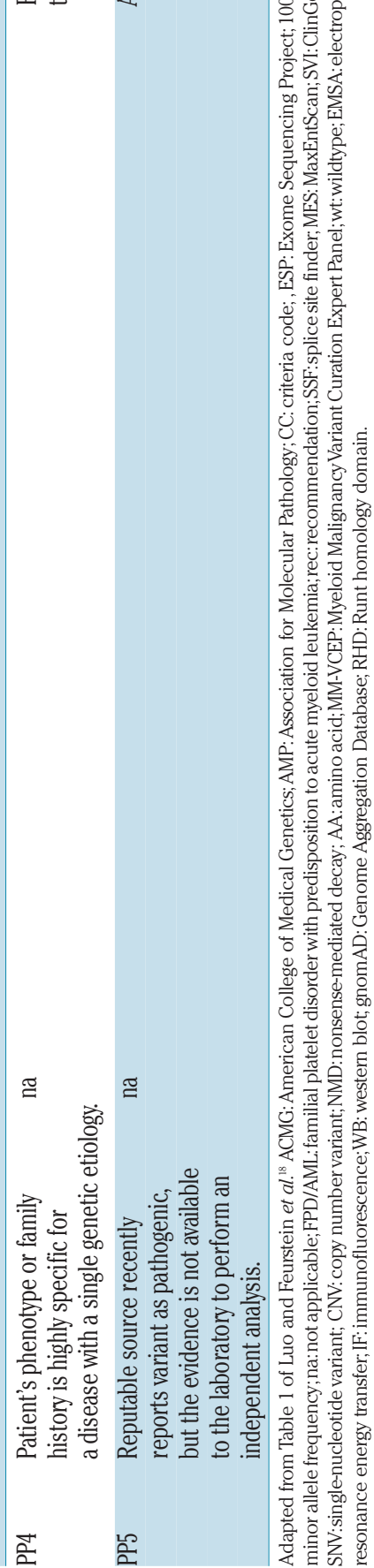


fibroblasts later confirmed the germline, nonsense RUNX1 variant. Her two sons and granddaughter also tested positive for the RUNX1 variant (Figure 1).

Similar to this case, most patients with FPD/AML will have a characteristic phenotype (Table 1) including mild to moderate thrombocytopenia with normal platelet size, platelet $\alpha$ or dense granule secretion defects and impaired platelet aggregation, particularly in response to collagen and epinephrine as well as a predisposition to hematologic malignancies. Although there is variability in disease onset in FPD/AML, ${ }^{3}$ development of a hematologic malignancy is common with a lifetime risk of $\sim 4 \%$ : AML and MDS are common, other malignancies occur less frequently (Table 1). ${ }^{19-23} \mathrm{FPD} / \mathrm{AML}$ has a high but incomplete

Table 3. Summary of RUNX1 variant examples with application of the Myeloid Malignancy Variant Curation Expert Panel criteria.

\begin{tabular}{|c|c|c|c|c|}
\hline Example No. & $\begin{array}{c}\text { RUNX1 variant } \\
\text { NM_001754 (isoform C) }\end{array}$ & $\begin{array}{l}\text { ClinVar } \\
\text { Assertion }\end{array}$ & Criteria & $\begin{array}{l}\text { MM-VCEP } \\
\text { classification }\end{array}$ \\
\hline 1 & c.610C >T (p.Arg204Ter) & PATH & PVS1, PM2, PS4_supporting, PP1 & PATH \\
\hline 2 & $\begin{array}{l}\text { c. } 314 \mathrm{~A}>\text { C p.(His105Pro) } \\
\text { c.315C }>\text { A p.(His105Gln) }\end{array}$ & VUS & $\begin{array}{c}\text { PM2, PP3, PS4_supporting, PM1_supporting, PM5_supporting } \\
\text { PS3, PM2, PP3, PM1_supporting }\end{array}$ & $\begin{array}{l}\text { LPATH } \\
\text { LPATH }\end{array}$ \\
\hline 3 & c.253C>A p.(His85Asn) & $\begin{array}{l}\text { CONF: } \\
\text { OMIM: PATH } \\
\text { Invitae: VUS }\end{array}$ & BS1, BS3, PP3 & LBEN \\
\hline 4 & $\begin{array}{c}\text { c. } 508+3 \text { delA } \\
\text { c. } 444 \mathrm{C}>\mathrm{T} \text { p. }(\text { Thr } 148=) \\
\text { c. } 1257 \mathrm{G}>\mathrm{A} \mathrm{p} .(\mathrm{Val} 419=)\end{array}$ & $\begin{array}{l}\text { PATH } \\
\text { Illumina: VUS } \\
\text { Invitae: LBEN } \\
\text { VUS }\end{array}$ & $\begin{array}{c}\text { PS3, PP1_strong, PM2, PP3, PS4_supporting } \\
\text { BP4, BP7 }\end{array}$ & $\begin{array}{l}\text { PATH } \\
\text { LBEN } \\
\text { VUS }\end{array}$ \\
\hline 5 & Copy number variant, deletion of exon 2 & & PS4, PP1_strong, PM2, PVS1_moderate & PATH \\
\hline 6 & c.1118C >A (p.Ser373Ter) & & PVS1_strong, PM2, PS4_supporting & LPATH \\
\hline
\end{tabular}

The five-tier ClinVar classification: PATH (pathogenic), LPATH (likely pathogenic),VUS (variant of uncertain significance), LBEN (likely benign), BEN (benign); CONF (conflicting interpretations in ClinVar); criteria from Luo and Feurstein et al..$^{18}$

RUNX1 NM_001754:c.610C>T, (p.Arg204Ter)

\section{I}

II

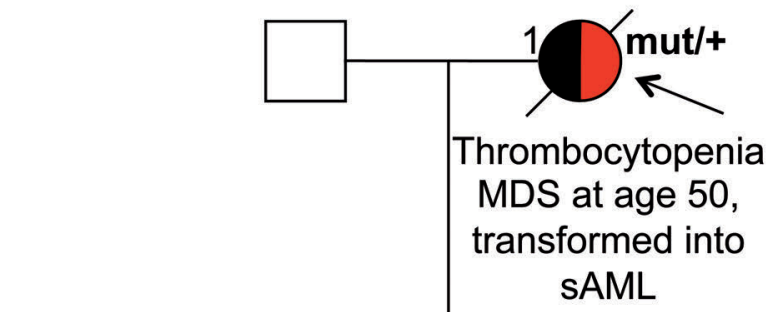

Figure 1. Family pedigree of a patient with acute myeloid leukemia, example 1 Germline RUNX1 NM_001754:c.610C>T, (p.Arg204Ter) showing typical autosomal dominant inheritance with other first and second-degree relatives with thrombocytopenia and/or myeloid malignancy (acute myeloid leukemia and myelodysplastic syndrome). mut: mutated; MDS: myelodysplastic syndrome; SAML: secondary acute myeloid leukemia; +: wildtype.

III

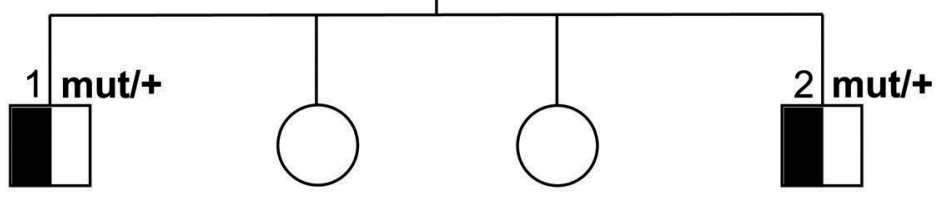

Thrombocytopenia

Thrombocytopenia

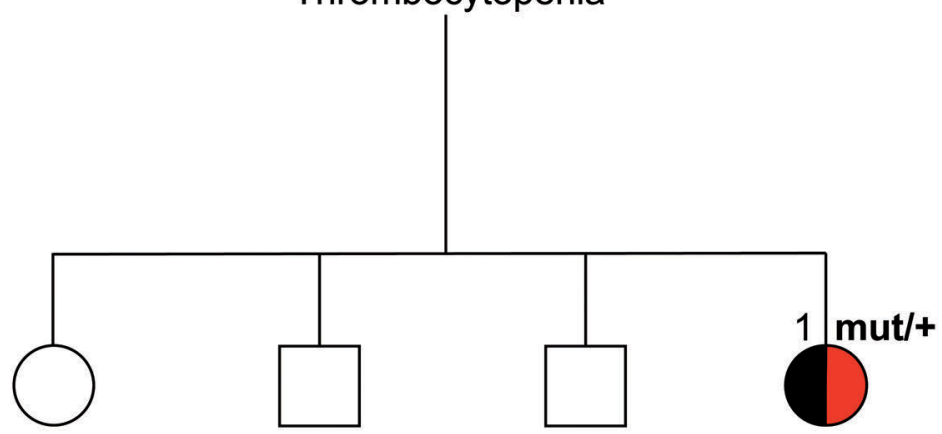

Thrombocytopenia

Childhood MDS 

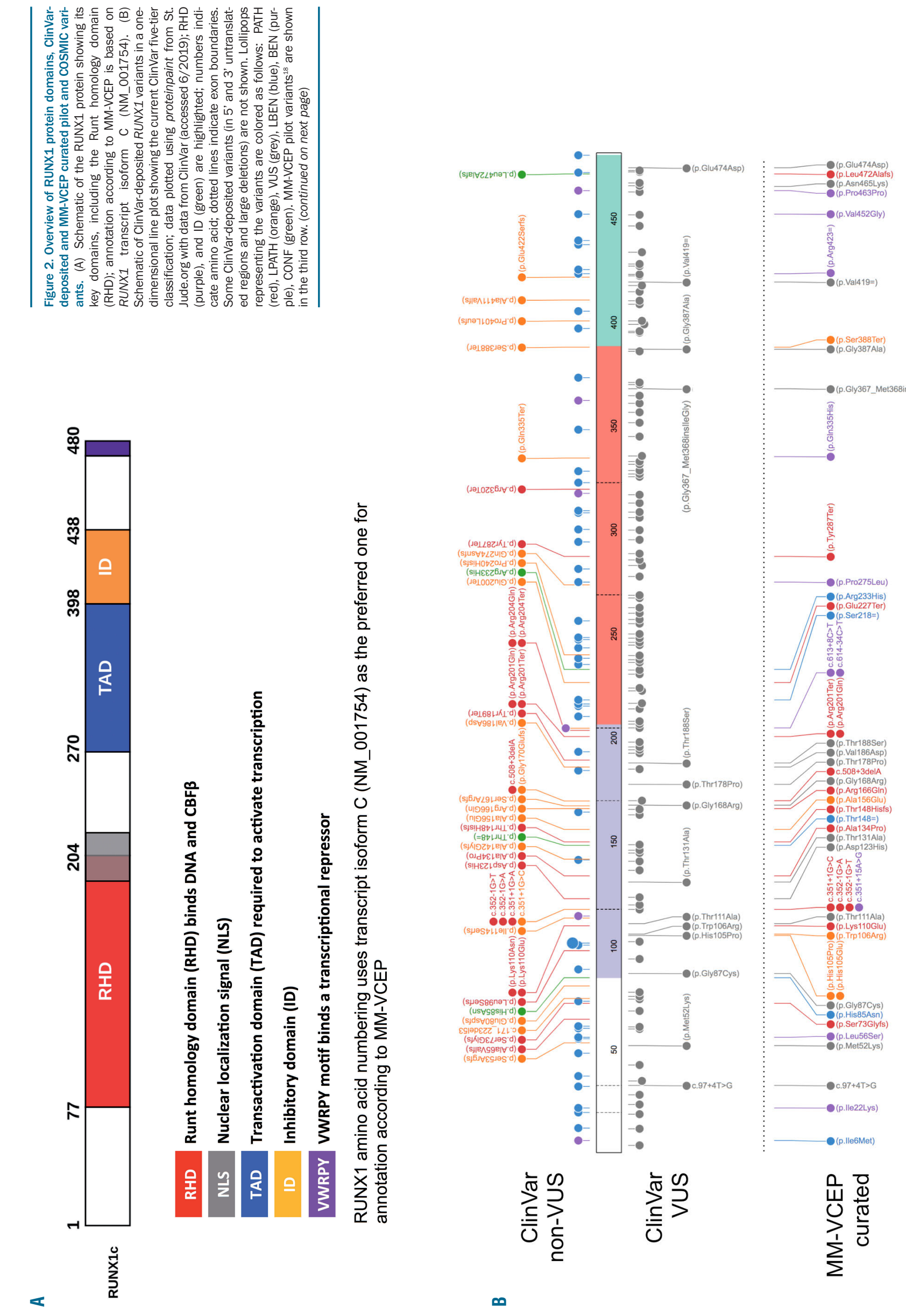

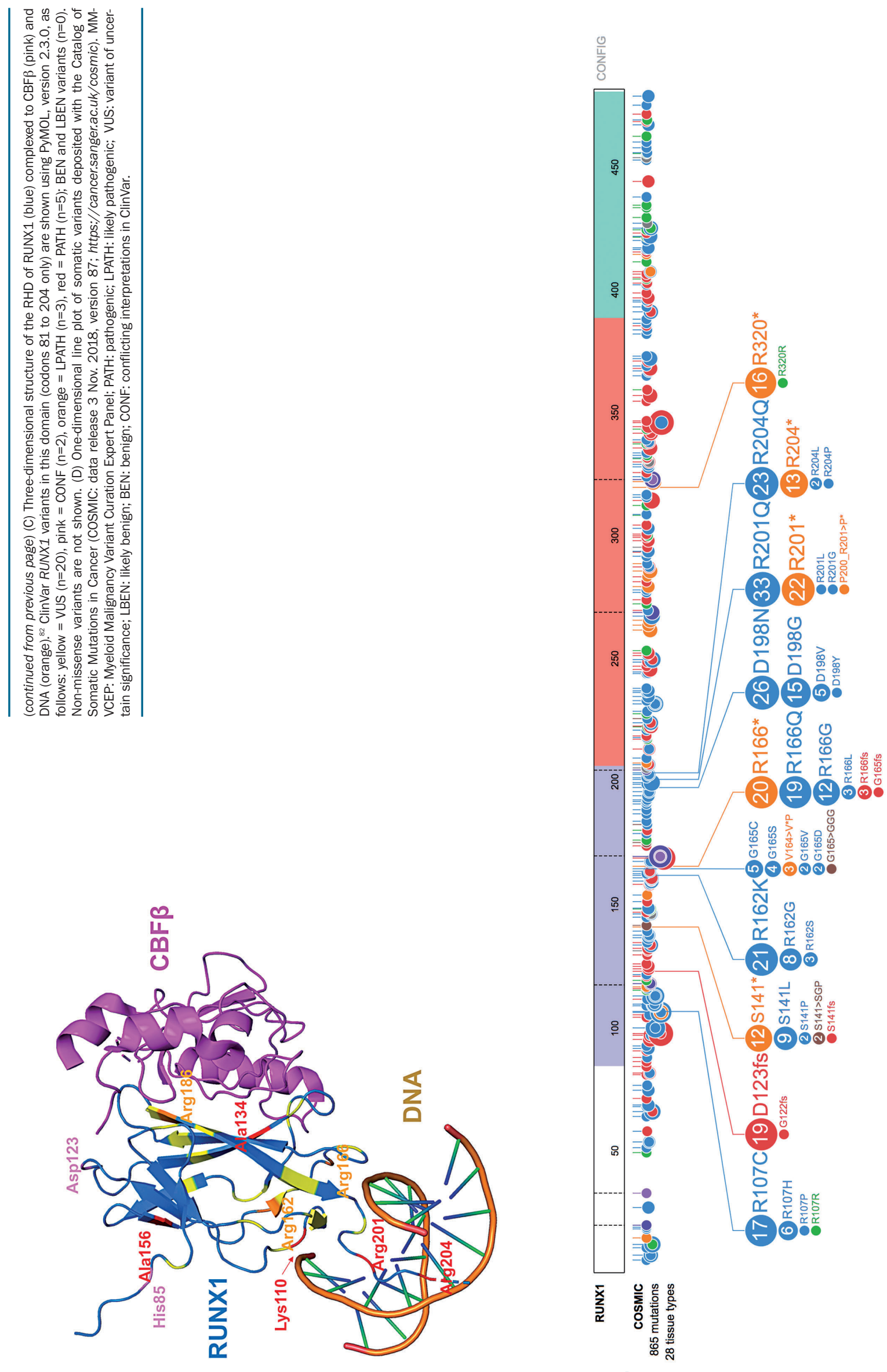
penetrance, with several affected individuals reported to have normal platelet counts or function. ${ }^{19,24}$ The nonsense mutation in this patient (p.Arg204Ter) is predicted to lead to nonsense-mediated decay of the RUNX1 mRNA transcript. RUNX1 is located on the long arm of chromosome 21 and is translated into three major isoforms, designated RUNX1A, RUNX1B, and RUNX1C, by using two different promoters and alternative splicing. All transcripts are expressed during hematopoietic differentiation and/or maintenance of normal bone marrow function. ${ }^{25-32}$ For variant annotation, the MM-VCEP utilizes the longest isoform, RUNX1C (NM_001754), as the default transcript, which includes all key domains such as the 128 amino acid (AA) long RUNT homology domain for DNA binding (RHD, AA 77-204), transactivation domain (TAD), inhibitory domain (ID) and the transcriptional repressor binding motif (VWRPY) and is most often used by clinical laboratories for $R U N X 1$ variant curation (Figure 2A). Germline variants have been reported throughout the gene in ClinVar with the majority currently classified as VUS (Figure 2B). The RUNX1 protein heterodimerizes through its RHD with CBF $\beta$ to form a master hematopoietic transcription factor (Figure 2C), which is essential for proliferation and differentiation of hematopoietic stem and progenitor cells, especially in the case of megakaryocytic differentiation. ${ }^{33-35}$ Somatic mutations commonly occur in RUNX1 (Figure 2D). According to $R U N X 1$-specific criteria (Table 2), ${ }^{18}$ the MM-VCEP applied the following codes (Table 3): PVS1 (nonsense variant predicted to undergo nonsense-mediated decay), PM2 (absence in all population databases), PS4_supporting (one proband meeting at least one of the RUNX1 phenotype criteria), PP1 (co-segregation with disease in the family, three meioses) and arrived at a consensus classification of PATH.

Example 2. Missense variants, p.(His105Pro) (LPATH with PM2, PP3, PS4_supporting, PM1_supporting, and PM5_supporting) and p.(His105GIn) (LPATH with PS3, PM2, PP3, and PM1_supporting)

Missense mutations in RUNX1 commonly occur in the RHD in somatic and germline contexts. ${ }^{36-38}$ Of 325 RUNX 1 ClinVar variants $122(37.5 \%)$ are missense, and currently in ClinVar, none in the RHD has been classified as BEN or LBEN (Figure 2C). When a novel missense variant is identified which has not been established as PATH or LPATH, it can be difficult to know whether the given change will affect protein function and explain the patient's phenotype. For example, two RUNX1 missense variants in the RHD (NM_001754:c.314A>C, p.(His105Pro); and NM_001754:c.315C>A, p.(His105GIn)) were considered during the pilot variant analysis. The former was initially classified as a VUS in ClinVar (Figure 2B), but subsequently revised to LPATH upon MM-VCEP review (Figures $2 \mathrm{~B}$, $3 \mathrm{~A}$ and 4). The conclusion of the LPATH assertion is based on the codes applied for this variant: PM2, PP3, PS4_supporting, PM1_supporting, and PM5_supporting (Table 3). Since the variant is completely absent from population databases, the MAF code PM2 is applied. For in-silico evaluation of missense variants, the MM-VCEP recommends using REVEL, a meta-predictor that combines 13 individual tools with high sensitivity and specificity, which has demonstrated the highest performance compared with individual tools or other ensemble methods. ${ }^{39-41}$ The computational prediction code PP3 is applica- ble to the p.His105Pro variant due to a high REVEL score of 0.953 (MM-VCEP defined $>0.75$ as the cutoff). The ClinVar submitter (SCV000807773.1) provided us with the patient's clinical data from their laboratory and the proband met at least one of the RUNX1 phenotype criteria (Table 1) which qualified for PS4_supporting. This example emphasizes the critical value of sharing internal laboratory data. There is only one meiosis in this family which is lower than the three required for the segregation code PP1. The MM-VCEP defined 13 residues in the RHD as the mutational hotspots for the PM1 code. In addition, variants in other parts of the RHD (AA 105-204) can have a reduced strength-level resulting in application of PM1_supporting. The last code PM5_supporting is applied on the p.(His105Pro) variant, because a different missense change p.(His105Gln) at the same residue has been classified as LPATH by the MM-VCEP (Table 3).

The codes PM2, PP3, PM1_supporting are also applicable to the p.(His105Gln) variant for the same reasons described. Furthermore, a strong pathogenic code PS3 is applied which contributes a significant weight to the final assertion. Transactivation assays of the p.(His105Gln) variant demonstrate altered transactivation $(<20 \%$ of wildtype) and secondary assays also indicate altered DNA binding and functional consequences in a mouse model ${ }^{42,43}$ manifested by disturbed myeloid differentiation and induction of a blast crisis or accelerated phase-like phenotype in mice. ${ }^{42}$ These variants highlight the importance of evaluating similar variants and the critical benefit of functional studies showing that variants whose clinical significance were initially uncertain can be subsequently clarified to provide more definitive clinical classification and minimize reporting of VUS. Moreover, these variants demonstrate the value of leveraging the information on one variant to help classify another and data sharing between laboratories (Table 3).

\section{Example 3. Missense variant, p.His85Asn (LBEN with} BS1, BS3, and PP3)

RUNX1 NM_001754:c.253C>A, p.(His85Asn) is a missense mutation located within the RHD, but not within the mutational hotspot region (AA 105-204), with conflicting interpretations of pathogenicity in ClinVar (Figure $2 \mathrm{~B}, \mathrm{C})$. Specifically, this variant had three submissions in ClinVar with two being PATH (submitted in 2002) and one being a VUS (submitted in 2018). The two 2002 submissions are from OMIM, which cited evidence from individual literature sources without a systematic curation process. Osato et al. reported an adult patient with AML carrying this variant. ${ }^{44}$ However, the germline nature of the variant was not definitively determined. This variant has also been reported in an infant diagnosed with transient myeloproliferative disorder and Down syndrome whose phenotype does not meet any of the RUNX1 phenotype criteria. ${ }^{45}$ After analysis and curation by the MM-VCEP using the RUNX1-specific classification rules, ${ }^{18}$ this variant was re-classified as $\mathrm{LBEN}$, meeting codes BS1 and BS3, despite meeting PP3 (Table 3). According to the penetrance, prevalence and genetic and allelic heterogeneity of RUNX1, MM-VCEP refined the RUNX1 specific MAF threshold for application of BS1 to $0.00015(0.015 \%)$. The highest MAF of the p.His85Asn variant is 0.00043 (8 out of 18,768 alleles) from the East Asian subpopulation in the Genome Aggregation Database (gnomAD) which is higher than the RUNX1- 
specific BS1 cutoff. Experimental studies have shown that this missense change displays normal transactivation activities (80-114\% of wildtype) and does not affect DNA binding, heterodimerization with $\mathrm{CBF} \beta$ or subcellular localization of the RUNX1 protein. ${ }^{44,46}$ Therefore, the strong benign functional evidence code, BS3, is applied. Although this variant disrupts KMT2A binding, which impairs proper H3K4 histone methylation, this is not a qualified functional assay based on the MM-VCEP RUNX1-specific PS3/BS3 rule. Moreover, another wellestablished BEN variant p.(Leu56Ser) also impairs KMT2A binding. ${ }^{46}$ Likely due to the location of the p.(His85Asn) variant within the RHD (Figure 2B), the REVEL score (0.852) of this variant is higher than the MM-VCEP defined 0.75 cutoff, ${ }^{18}$ which results in the variant meeting a conflicting PP3 code. However, combining the BS1, BS3 and PP3 codes, a final assertion of LBEN is made based on a Bayesian classification framework. ${ }^{15}$ Given that His85 is located away from binding interfaces in the three-dimensional structure (Figure 3B), it seems reasonable that variants at this position are LBEN.

This example highlights the value of functional studies in the context of the MM-VCEP variant curation and shows that substantive corrections of variant annotation may occur upon application of ClinGen MM-VCEP rules. ${ }^{18}$ Implicit in this process is the expectation that as knowledge about FPD/AML improves with more functional or family data becoming available, the MM-VCEP rules are subject to revision so that annotation of clinical variants will become more accurate (Figure 4). ClinVar variant classifications such as VUS or those with conflicting interpretations may thus evolve to more diagnostic certainty. ${ }^{47,48}$

Example 4. Synonymous/intronic/non-coding variants, c.508+3delA (PATH with PS3, PP1 strong, PM2, PP3, PS4 supporting), p.Thr148= (LBEN with BP4, BP7) and p.Val419= (VUS with no codes)

$R U N X 1$ variants affecting canonical splice positions \pm 1 or 2 at intron-exon boundaries are expected to disrupt

A
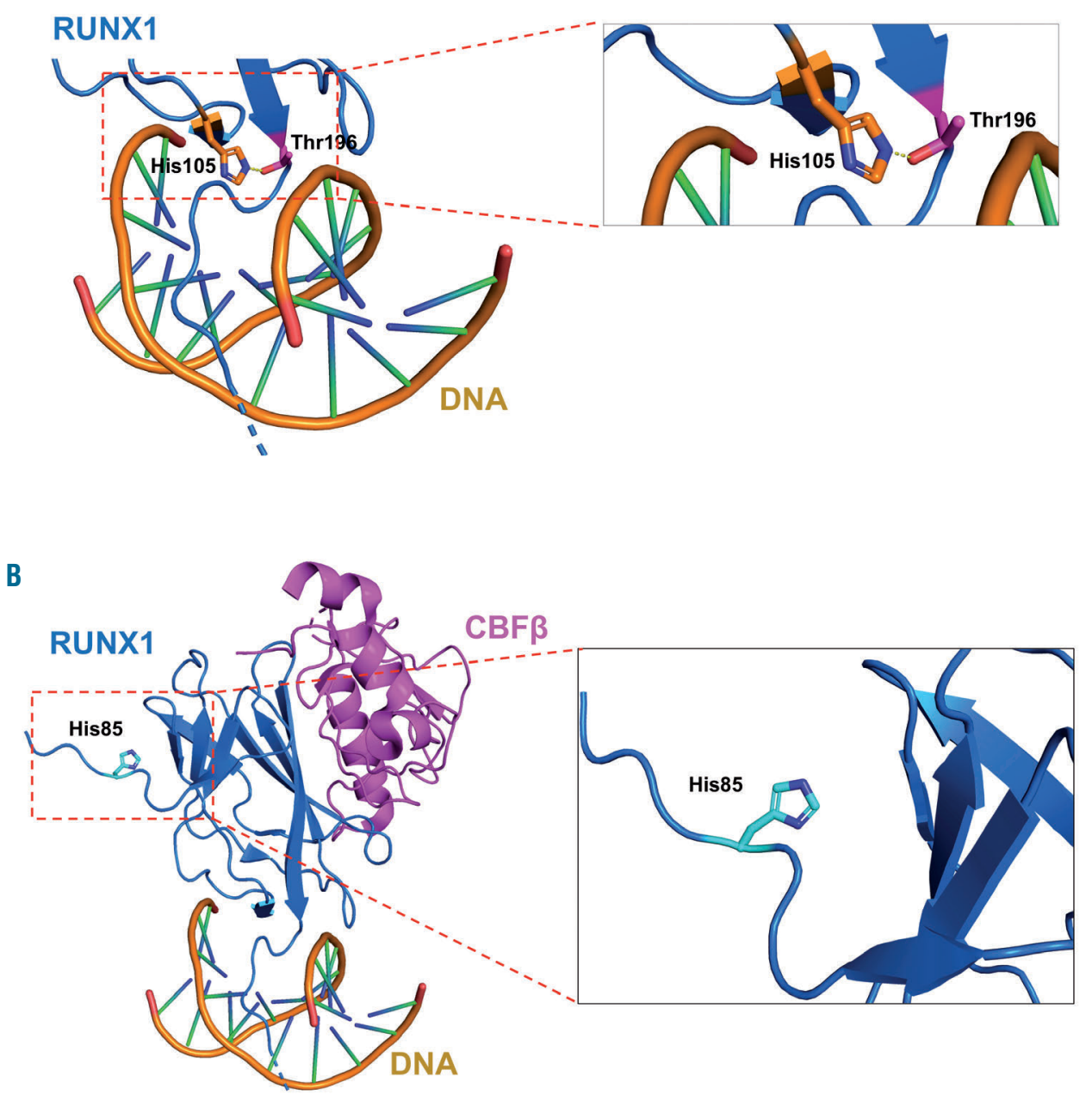

Figure 3. Three-dimensional structure of RUNX1 missense variants His105 and His85 considered as examples 2 and 3 . (A) RUNX1 His 105 is important functionally due to its location and thus involvement in DNA binding and close interaction with Thr196 by hydrogen bonding. Thr196 is a hotspot residue known to be critical. This structure-function relationship further supports classification of His105 variants as likely pathogenic (LPATH). (B) RUNX1 His85 is located close to the start of the Runt homology domain in a linker region, and is located far from the DNA binding surface. It is not involved in the core $\beta$-barrel structure and does not show any interactions, further supporting its classification as likely benign (LBEN). Structure of RUNX1 complexed to DNA and CBF $\beta$ (https://www.rcsb.org/structure/1H9D) ${ }^{82}$ and plotted using PyMOL version 2.3.0. 
splicing, leading to protein dysfunction (see Online Supplementary Table S3 of reference by Luo and Feurstein ${ }^{18}$ ). All of the three canonical splicing site variants in the pilot set were classified as PATH or LPATH. More challenging, however, is the consideration of synonymous/intronic/non-coding variants which may result in cryptic splice site activation, and/or enhancement or repression of adjacent canonical splice sites. For example, the intronic NM_001754:c.508+3delA variant has been reported in a single family with disease segregation (8 meioses, PP1_strong). Several family members were diagnosed with thrombocytopenia, aspirin-like platelet aggregation defects, and dense granule abnormalities. ${ }^{49}$ This variant is absent from population databases (PM2) and both splicing predictors (MaxEntScan and SpliceSiteFinder $)^{50,51}$ predict a significant decrease in the score of the canonical splice site (PP3). Moreover, experimental reverse transcriptase polymerase chain reaction studies (PS3), using RNA derived from two affected family members, were performed and indicate the creation of a novel cryptic splice site 23 nucleotides upstream of the normal splice site resulting in a frameshift p.(Arg162fs*177), and the transcript is predicted to undergo nonsense-mediated decay. ${ }^{49}$ Combining all of these codes, a final assertion of PATH is given by the MM-VCEP (Table 3).

BP7 is a benign code specifically designed to evaluate synonymous/intronic/non-coding variants in the ACMG/AMP framework. BP7 can be applied if computational evidence suggests no impact on splicing, and the nucleotide is not conserved. The ClinVar variant with conflicting interpretations in ClinVar, NM_001754:c.444C>T, p.(Thr148=), has been classified as LBEN by the MM-VCEP using BP7 and the benign in silico prediction code, BP4 (Table 3). This nucleotide change is predicted to have no impact on splicing and it is also not conserved (phyloPscore: -4.3832, below the MM-VCEP-specified threshold of $<0.1^{18}$ ). Clinical data from seven individuals with this variant were acquired from the original ClinVar submitter (SCV000761123.1) and revealed that none of the probands met any of the RUNX1 phenotypic criteria. ${ }^{18}$ Currently, only two RUNX1 variants have been reported to display an abnormal splicing effect as demonstrated by RNA assays. ${ }^{11,49}$ The potential effects of other splicing variants rely solely on in silico predictions. Although there is robust effort in consideration of algorithms to predict the effects of splicing variants, these algorithms require further evaluation. Indeed, we know of only limited experimental data within the RUNX1 gene specifically to test these tools. Accordingly, the synonymous variant, NM_001754:c.1257G>A, p.(Val419=) is predicted to create alternative splice acceptor sites, but is not expected to abolish any existing consensus sites, as it is too far away from either end of the exon. Due to this in silico prediction result, none of the PP3/BP4 and BP7 codes can be assigned, and the classification of this variant remains a VUS. Further resolution of the significance of this variant could be obtained through parental testing, and/or RNAsequencing data.

Example 5. Copy number variants, deletion of exon 2 (PATH with PVS1_moderate, PM2, PS4, and PP1_strong)

Not infrequently, patients with FPD/AML have been reported to have copy number variants resulting in intragenic deletions of RUNX1.52 As part of our pilot cohort, we evaluated several probands with copy number vari-

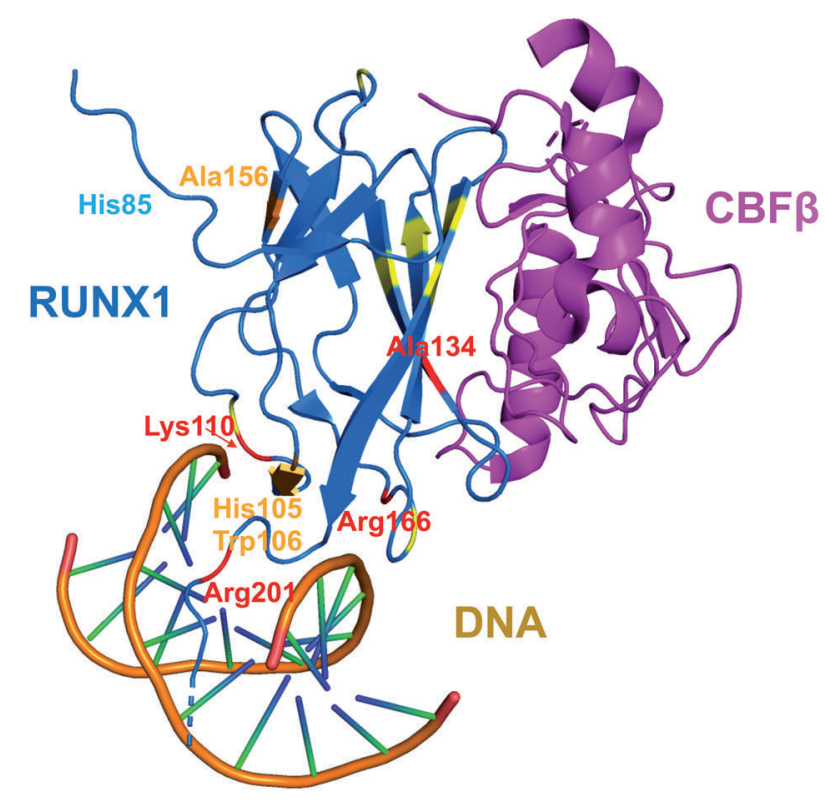

Figure 4. Three-dimensional structure of MM-VCEP-classified RUNX1 missense variants in the RHD. Variants in the RUNX1 RHD (blue) are shown highlighting the PATH, LPATH, and LBEN missense variants curated according to ClinGen MM-VCEP rules. PATH (red, $n=4$ ), LPATH (orange, $n=4$ ) and LBEN (cyan, $n=1$ ) variants are found proximate to key interaction domains of RUNX1 with DNA or its binding partner CBF $\beta$ (pink). VUS missense variants are shown in yellow $(n=7)$. Non-missense variants and variants outside the RHD are not shown. (PyMOL version 2.3.0; structural data https://www.rcsb.org/structure/1H9D ${ }^{82}$ ). MM-VCEP: Myeloid Malignancy Variant Curation Expert Panel; RHD: Runt homology domain; PATH: pathogenic; LPATH: likely pathogenic; VUS: variant of uncertain significance; LBEN: likely benign; BEN: benign; CONF: conflicting interpretations in ClinVar.

ants which at a minimum include RUNX1 exon 2 deletion; data from two cases are shown (Figure 5). The analysis of copy number variants by using next-generation sequencing and/or single nucleotide polymorphism microarrays is particularly challenging because the breakpoints are often not captured in the sequenced regions or the microarray resolution defines only a range for the chromosomal location of the breakpoint, respectively, and thus the nucleotide level breakpoint may remain unknown. It can, therefore, be difficult to know the effect of partial gene/exon deletions, such as if the deletion is in- or out-of-frame, the latter of which may also lead to the introduction of a premature stop codon. Nevertheless, partial or whole gene deletion of RUNX1 is expected to result in haploinsufficiency of the RUNX1 protein. Although MM-VCEP rules did not include recommendations for the formal classification of copy number variants, ${ }^{18}$ several points should be noted. First, evaluation of the reference Database of Genomic Variants (http://dgv.tcag.caldgv/app/home) shows that copy number variants affecting RUNX1 do not appear to be frequent. ${ }^{18}$ Second, annotation of the specific breakpoints of these intragenic deletions may not always be possible, given that whole genome sequencing is not typically performed. Since contiguous exon deletion is a common pathogenic disease mechanism, it is imperative that laboratories performing germline testing for RUNX1 use concurrent microarray testing, develop appropriate next-generation sequencing bioinformatics pipelines, or use alternate molecular techniques, such as quantitative polymerase chain reaction and multiplex ligation-dependent probe amplification, to screen for and exclude copy loss, 
A

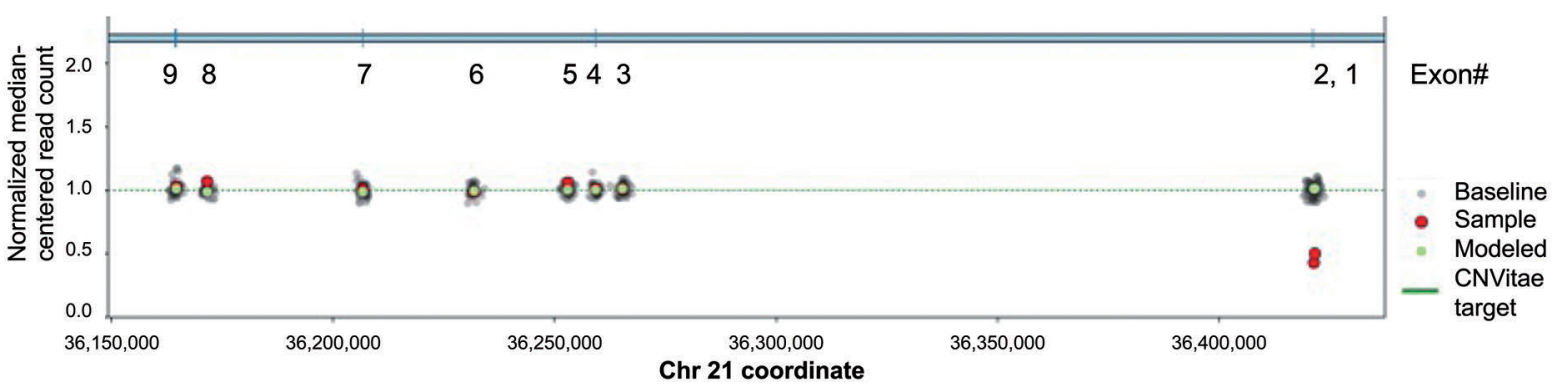

B

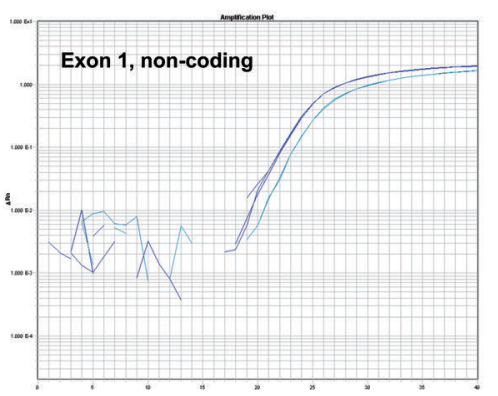

Cycle \#

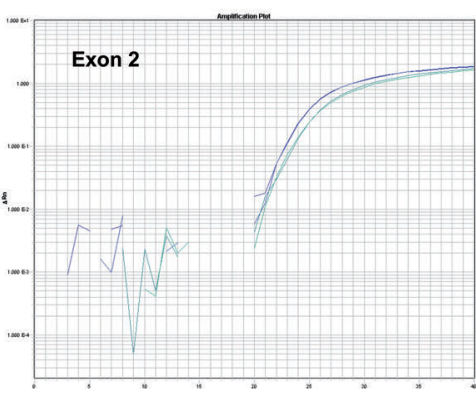

Cycle \#

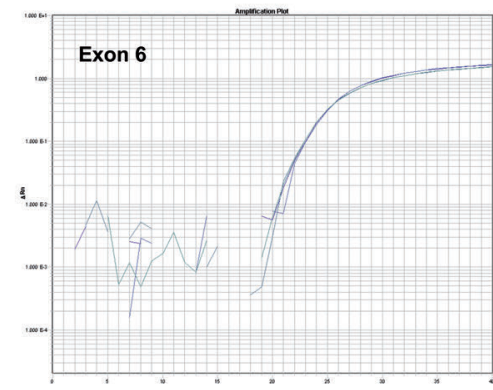

Cycle \#

Figure 5. Copy number variant, deletion of exons of RUNX1. Testing for germline variants should include evaluation for copy number variants (CNV). (A; B) Clinical data from two different patients showing copy loss of RUNX1 exons 1 and 2; one proband was identified by next-generation sequencing (A) and the other by single nucleotide polymorphism array analysis (data not shown), and confirmed by quantitative polymerase chain reaction (qPCR) (B). For the latter patient, qPCR confirmation showed heterozygous deletion of exons 1 and 2 with no loss in other exons (exon 6 only shown). CNV are difficult for annotation as breakpoints of the deletion may not be captured and thus, whether the deletion is in- or out-of-frame may not be known without whole genome sequencing. Testing of a germline sample (e.g. fibroblasts) is preferred to blood or bone marrow for CNV evaluation, as somatic copy loss may also occur in the tumor context.

Table 4. Variant details for example 6 from genome and exome sequencing, RNA-sequencing and karyotype analysis.

\begin{tabular}{ll}
\hline Structural & KMT2A, NM_005933, 11q23 (chr11:118347001-118353900) partial tandem duplication exons 4-8, predicted to be in-frame \\
SNVs and indels & IDH1, NM_005896:c.394C >T, p.(Arg132Cys), VAF 50\% \\
& PHF6, NM_001015877:c.860G >T, p.(Gly287Val), VAF 95\% \\
& RUNX1, NM_001754:c.1118C >A, (p.Ser373Ter), VAF 50\% \\
\hline Copy number variants & PALB2, heterozygous exon 7 copy loss, NM_02467, chr16p12.2(23637272_23637800)x1 for hg19 \\
\hline
\end{tabular}

Chr: chromosome; VAF: variant allele frequency.

as sequencing for single nucleotide variants and indels alone is insufficient for comprehensive germline evaluation. For the two cases herein (Figure 5), although we do not know the specific breakpoints for each, the common deletion of at least exon 2 allows us to apply the following codes: PVS1_moderate, PM2, PS4 (4 probands: 3 with chronic thrombocytopenia and 1 with AML), PP1_strong (7 meioses) to arrive at a PATH classification (Table 3).

Example 6. Late truncation variant, (p.Ser373Ter) (LPATH with PVS1_strong, PM2, and PS4_supporting)

A 14-year old male with a non-contributory family history presented with malaise, poor appetite, night sweats, and intermittent fever of about 1-month duration, thrombocytopenia $\left(27 \times 10^{9} / \mathrm{L}\right)$, and subsequent bone marrow biopsy showed AML (PS4_supporting). After whole genome and exome sequencing on paired tumor and germline samples, along with RNA-sequencing (directed for recurrent fusion identification), his leukemia sample was shown to be $R U N X 1$-mutated (NM_001754:c.1118C>A, (p.Ser373Ter)), hypodiploid with Y chromosome loss, without chromosomal fusions, and loss of heterozygosity, but had additional mutations including an intragenic heterozygous deletion of one copy of exon 7 of PALB2 (Table 4).

Similar to Example 1, this RUNX1 (p.Ser373Ter) variant is a nonsense mutation; however, it is not predicted to undergo nonsense-mediated decay, but rather is expected to generate a truncated protein without part of the TAD, ID and the VWRPY motif (Figure 2A). From a computational and predictive perspective, a PVS1_strong code is assigned following the PVS1 decision tree for null or truncating variants in RUNX $1 .{ }^{18}$ The variant is absent from the gnomAD and other population databases (with confirmed $>20 \mathrm{x}$ sequencing coverage at this position in gnomAD). Given the variant's absence from population databases and adequate sequencing coverage of the region, a PM2 code is assigned. Although no additional evidence for the other categories (functional, segregation, de novo and allelic data) are available, this variant can be classified as LPATH (PVS1_strong, PM2, PS4_supporting). It is of interest to note that the somatic alterations reported in the diagnostic leukemia sample included partial tandem duplication of KMT2A and a single nucleotide varia- 
tion in PHF6. Alterations in these two genes have been reported as cooperating events seen in leukemias from patients with germline RUNX1 mutations..$^{37,53}$

Importantly, if consideration is given to the mutations found in the leukemic cells in isolation, one cannot determine the germline or somatic origin of the variants reported. This is the case for most of the 'tumor-only' analyses being performed in many clinical laboratories. Without paired analysis of true germline tissue (e.g. cultured skin fibroblasts), such studies cannot definitively identify germline variants. In this case, the KMT2A partial tandem duplication and single nucleotide variation in PHF6 and RUNX1 could be tumor-drivers in the AML. However, given the sequencing data, including the variant allele frequency, both the RUNX1 mutation and the $P A L B 2$ exon 7 intragenic deletion could be germline variants. A detected variant allele frequency approaching $50 \%$ or $100 \%$ in the tumor may indicate potential germline origin ${ }^{1}$ with either an intact wildtype allele or loss of heterozygosity, respectively. However, a high variant allele frequency cannot reliably serve as a proxy for testing of a true germline source. Therefore, if there is concern that a variant could be constitutional, testing of true germline material is critical. ${ }^{1}$

\section{Discussion}

Kindreds with FPD/AML were first reported by Luddy et al. in $1978^{54}$ and phenotypically well-described as having a bleeding diathesis and myeloid neoplasia by Dowton et al. in 1985..$^{5,56}$ Subsequent linkage analysis identified $R U N X 1$ as the candidate gene at chromosome $21 \mathrm{q} 22,{ }^{11}$ and mutations were detected in FPD/AML families in $1999 .{ }^{11}$ Since these initial early reports, routine clinical testing for RUNX1 gene mutations is now commonplace for the evaluation of somatic and germline disease in patients with myeloid neoplasms and thrombocytopenia.

In general, RUNX1 variants include single nucleotide variations and indels, such as missense, nonsense, frameshift, and splice site variants, and copy number variations such as whole-gene and intragenic deletions. RUNX1 is also frequently mutated somatically in AML and often the partner of various translocations resulting in gene fusions, such as $\mathrm{t}(8 ; 21)(\mathrm{q} 22 ; \mathrm{q} 22)$ RUNX1RUNX1T1 $1{ }^{57,58}$ To date, fusions of RUNX1 have not been reported in the germline context, and most germline RUNX1 variants are unique, ${ }^{24}$ although some have been rarely seen in unrelated families. Given the limited data on rare variants, the clinical annotation of new variants remains challenging. The MM-VCEP was convened by ASH/ClinGen (Online Supplementary Figure S1) to develop rules for curating gene variant causing predisposition to myeloid neoplasia (Table 2). In this review, we describe the classification of six variant examples (Table 3 ) using the gene- and disease-specific rule modifications of the original ACMG/AMP 2015 framework. ${ }^{14}$ Several points should be made.

First, it is critical to ensure that genomic testing intended to assess a germline predisposition is performed on a definitive germline sample because malignant hematologic diseases involve the peripheral blood and bone marrow, and somatic variants in these diseases can confound variant interpretation if an inappropriate sample is used.
Here, in keeping with our MM-VCEP rules, cultured skin fibroblasts (gold standard, albeit invasive, costly, and time-consuming), cultured bone marrow mesenchymal stromal cells or DNA from hair roots are appropriate sources. ${ }^{59,60}$ Alternatively, confirmation of the germline nature of a variant can be achieved by demonstrating its presence in two or more related individuals. The possibility of sample contamination by malignant cells is significant and consequently, peripheral blood, bone marrow, saliva, buccal swabs, DNA from paraffin blocks and even fingernails, which can contain monocytes, are inappropriate samples for germline testing. In some institutions, laboratories may accept $T$ cells, enriched via flow cytometry sorting or column-based magnetic cell separation, as a germline sample for testing. It is important to recognize that some somatic alterations may occur early in hematopoietic stem and progenitor cells with multilineage potential to differentiate into $\mathrm{T}$ cells, ${ }^{61}$ as recent single-cell studies have confirmed. ${ }^{62-65}$ Thus, if $\mathrm{T}$ cells are used, the possibility that a detected variant may be somatic should still be considered. Once a variant is confirmed to be germline in a proband, however, additional testing for the known variant in related family members can be performed on any tissue source.

Second, we should keep an open mind about diseasecausing alleles and the type of variants that may be seen and thus, we advocate for a broad testing approach. For example, in some laboratories, non-coding variants are automatically filtered as part of bioinformatic pipelines and may thus be omitted from subsequent review and interpretation. Recently, however, synonymous variants ${ }^{66}$ in the GATA2 gene, another gene predisposing to myeloid malignancy, were reported in addition to the known pathogenic deep intronic variants of an enhancer region of GATA2. ${ }^{67}$ In ANKRD26, variants of the 5' untranslated region cause disease. ${ }^{68,69}$ Furthermore, copy number alterations may not be assessed in somatic tumor testing panels. As diagnosticians, it is important to think broadly when analyzing genomic information for germline pathogenic variants. Given that these are rare diseases, we should not inadvertently exclude disease mechanisms and/or specific classes of mutations. For example, in case 4, some variants may remain as VUS until additional functional or familial segregation data become available for reclassification. ${ }^{47,48}$

Third, definitive annotation of variants by one institution will likely remain challenging. However, consistent application of MM-VCEP rules with ClinVar data deposition and thus inter-laboratory correspondence can significantly improve the accuracy and consistency of variant curation. In this regard, examples 2, 3, and 5 show how leveraging shared genomic and phenotypic data can be helpful to clarify VUS. We therefore advocate that clinical variant data be deposited into ClinVar. Specifically, laboratories offering germline testing should modify their test requisition forms to indicate that de-identified phenotype and variant data will be deposited into ClinVar as part of ongoing quality assurance and improvement efforts (https://WwW.clinicalgenome.org/share-your-data/laboratories/). ${ }^{70,71}$ Additional details of the ClinVar deposition process are included in Online Supplementary Figure S1.

Fourth, RUNX1 variant curation will improve as more is understood about the disease and gene through functional and family studies. Currently, variant annotation remains a challenging task, because of limited data for 
determining the functional effect of a given variant change, despite methods of engineering variants for functional assessment. ${ }^{72-74}$ Early studies in Speck's laboratory on RUNX1 showed the significance of key residues in the RHD of RUNX1 by performing alanine scanning mutagenesis. ${ }^{75,76}$ However, these early approaches are limited in that not every combination of nucleotide change was explored. By contrast, recent high-throughput functional genomic methods, ${ }^{77}$ known as deep mutational scanning, utilize large-scale approaches to mutate every nucleotide of a gene, permitting one to test the functional consequence of all single nucleotide variations. This has, for example, been recently demonstrated for $B R C A 1 .^{78}$ Additionally, systematic mutagenesis of PTEN has provided a wealth of functional data to inform the classification of PTEN variants, ${ }^{79}$ in conjunction with published rules developed by the PTEN-VCEP. ${ }^{80}$ In the future, focused functional assays targeting specific VUS ${ }^{16}$ and deep mutational scanning of genes should contribute to variant curation to resolve VUS.

Fifth, while functional testing of every given genomic variant is possible, it can be costly and difficult to do for every clinically significant gene. In this regard, family studies can aid in the classification of VUS. By systematically evaluating disease segregation in family members with paired genotyping for a known variant, accurate classification of a given variant can be achieved. For example, a recent study showed that this family-based method for variant classification can resolve a VUS classification more frequently than other traditional approaches can. ${ }^{7,81}$ For rare diseases, such as FPD/AML, detailed pedigree and segregation analyses can be incredibly informative, and clinicians should be encouraged to test family members when possible, seeking help from local genetic counselors and/or geneticists as needed. ${ }^{81}$ Hematologists and oncologists need to consistently take a detailed family and genetic history.

\section{Summary}

RUNX1 germline mutations associated with FPD/AML are key events in myeloid neoplasms, thrombocytopenia and leukemogenesis and represent a model of a germline gene disorder with pathogenic variants predisposing to myeloid and (to a lesser extent) lymphoid malignancies. ${ }^{36}$ Providing an accurate clinical and pathologic variant interpretation for genomic variants detected in routine laboratory testing will remain critical for the provision of appropriate clinical care, including genetic counseling for the index patient and their at-risk relatives and donor-selection, in some cases benefiting from stem cell transplantation.

The ClinGen MM-VCEP variant interpretation process requires a detailed understanding of the biological and functional properties of RUNX1 and disease phenotype. Here, we demonstrate the process for sequence variant interpretation of six variant examples. By introducing and thus standardizing genomic variant interpretation, we hope to improve patients' care, identify VUS that may benefit from directed research and encourage sharing of internal laboratory data to resolve uncertainty. In doing so, the MM-VCEP rules may ensure optimal insurance coverage for appropriate genomic testing and screening of family members, and ensure appropriate reimbursement for clinical laboratories. Overall, the ASH/ClinGen collaboration resulting in the first set of modified criteria for germline RUNX1 variants should improve clinical care and recommendations for FPD/AML patients.

\section{Acknowledgments}

The Variant Curation Expert Panel (VCEP) thanks the Clinical Genome Resource (ClinGen) Sequence Variation Interpretation Working Group as well as the Executive Committee of the Hereditary Cancer Clinical Domain Working Group. The variant curation rules discussed in this publication were generated by the American Society of Hematology (ASH) in collaboration with Baylor College of Medicine and the University of North Carolina, National Institutes of Health (NIH)-funded Clin Gen grant award recipients. The NIH supported this work through: U41HG009649 (to XL, and SEP) and U41HG009650 (to SM and JER), and the $2018 \mathrm{NIH} / \mathrm{NCI}$ Leukemia SPORE DRP award (P50CA100632-16, project 00007529) (C.D.D.).

The ASH-ClinGen Myeloid Malignancy MM-VCEP collaborative group includes co-authors (DW and LAG as co-chairs; $X L, S F, S M, C K$ and DEP), and others, as follows: ${ }^{18}$ Christopher Porter (Emory University, USA), Sarah Jackson (GeneDx, USA), Sioban Keel (University of Washington, USA), Michael Chicka (Prevention Genetics, USA), Anna Brown (Center for Cancer Biology, Australia), Anupriya Agarwal (Oregon Health \& Science University, The Knight Cancer Institute, USA), Minjie Luo (Children's Hospital of Philadelphia, USA), Zejuan Li (Houston Methodist Institute for Academic Medicine, USA), Justyne E. Ross (University of North Carolina, USA), Panagiotis Baliakas (Uppsala University, Sweden), Courtney D. DiNardo (UT MD Anderson Cancer Center, USA), Alison Bertuch (Baylor College of Medicine, USA), Nikita Mehta (Mayo Clinic, USA), Thomas Vulliamy (Queen Mary University of London, UK), Ying Wang (BioReference, USA), Kim Nichols (St Jude Children's Research Hospital, USA), Luca Malcovati (University of Pavia \& S. Matteo Hospital, Italy), Michael Walsh (Memorial Sloan Kettering Cancer Center, USA), Lesley Rawlings (Centre for Cancer Biology, Australia), Shannon McWeeney (Oregon Health \& Science University, USA), Jean Soulier (Hopital Saint-Louis and University de Paris, France), Anna Raimbault (INSERM U1016, Institut Cochin, France), Mark Routbort (UT MD Anderson Cancer Center, USA), Living Zhang (Memorial Sloan Kettering Cancer Center, USA), Gabriella Ryan (American Society of Hematology, USA), Nancy Speck (Abramson Family Cancer Research Institute, USA), and Sharon E. Plon (Baylor College of Medicine, USA). 


\section{References}

1. Drazer MW, Kadri S, Sukhanova M, et al. Prognostic tumor sequencing panels frequently identify germ line variants associated with hereditary hematopoietic malignancies. Blood Adv. 2018;2(2):146-150.

2. Churpek JE, Pyrtel K, Kanchi KL, et al. Genomic analysis of germ line and somatic variants in familial myelodysplasia/acute myeloid leukemia. Blood. 2015;126(22): 2484-2490.

3. Churpek JE, Bresnick EH. Transcription factor mutations as a cause of familial myeloid neoplasms. J Clin Invest. 2019;129(2):476488.

4. Arber DA, Orazi A, Hasserjian R, et al. The 2016 revision to the World Health Organization classification of myeloid neoplasms and acute leukemia. Blood. 2016;127 (20):2391-2405

5. Swerdlow SH CE, Harris NL, Jaffe ES, Pileri $\mathrm{SA}$, Stein $\mathrm{H}$, Thiele J. WHO Classification of Tumours of Haematopoietic and Lymphoid Tissues, 2017.

6. Kohane IS, Masys DR, Altman RB. The incidentalome: a threat to genomic medicine. JAMA. 2006;296(2):212-215.

7. Shirts BH, Pritchard CC, Walsh T. Familyspecific variants and the limits of human genetics. Trends Mol Med. 2016;22(11):925934.

8. Beri-Dexheimer $M$, Latger-Cannard V, Philippe C, et al. Clinical phenotype of germline RUNX1 haploinsufficiency: from point mutations to large genomic deletions. Eur J Hum Genet. 2008;16(8):1014-1018.

9. Schlegelberger B, Heller PG. RUNX1 deficiency (familial platelet disorder with predisposition to myeloid leukemia, FPDMM). Semin Hematol. 2017:54(2):75-80.

10. Sood R, Kamikubo Y, P. L. Role of RUNX1 in hematological malignancies. Blood. 2017;129(15):2070-2082. Erratum in: Blood. 2018;131 (3):373.

11. Song WJ, Sullivan MG, Legare RD, et al. Haploinsufficiency of CBFA2 causes familial thrombocytopenia with propensity to develop acute myelogenous leukaemia. Nat Genet. 1999;23(2):166-175.

12. Landrum MJ, Lee JM, Riley GR, et al. ClinVar: public archive of relationships among sequence variation and human phenotype. Nucleic Acids Res. 2014;42 (Database issue):D980-985.

13. Rehm HL, Berg JS, Brooks LD, et al. ClinGen--the Clinical Genome Resource. N Engl J Med. 2015;372(23):2235-2242.

14. Richards S, Aziz N, Bale S, et al. Standards and guidelines for the interpretation of sequence variants: a joint consensus recommendation of the American College of Medical Genetics and Genomics and the Association for Molecular Pathology. Genet Med. 2015;17(5):405-424

15. Tavtigian SV, Greenblatt MS, Harrison SM, et al. Modeling the ACMG/AMP variant classification guidelines as a Bayesian classification framework. Genet Med. 2018;20(9): 1054-1060

16. Brnich SE, Rivera-Munoz EA, Berg JS. Quantifying the potential of functional evidence to reclassify variants of uncertain significance in the categorical and Bayesian interpretation frameworks. Hum Mutat. 2018;39(11):1531-1541.

17. Rivera-Munoz EA, Milko LV, Harrison SM, et al. ClinGen Variant Curation Expert Panel experiences and standardized processes for disease and gene-level specification of the ACMG/AMP guidelines for sequence vari- ant interpretation. Hum Mutat. 2018;39 (11):1614-1622.

18. Luo X, Feurstein S, Mohan S, et al. Genespecific criteria for germline RUNX1 variant curation: recommendations from the ClinGen Myeloid Malignancy Variant Curation Expert Panel. Blood Adv. 2019;3 (20):2962-2979

19. Owen CJ, Toze CL, Koochin A, et al. Five new pedigrees with inherited RUNX1 mutations causing familial platelet disorder with propensity to myeloid malignancy. Blood. 2008;112(12):4639-4645.

20. Shiba N, Hasegawa D, Park MJ, et al. CBL mutation in chronic myelomonocytic leukemia secondary to familial platelet disorder with propensity to develop acute myeloid leukemia (FPD/AML). Blood. 2012;119(11):2612-2614.

21. Linden T, Schnittger S, Groll AH, Juergens H, Rossig C. Childhood B-cell precursor acute lymphoblastic leukaemia in a patient with familial thrombocytopenia and RUNX1 mutation. Br J Haematol. 2010;151(5):528-530

22. Toya T, Yoshimi A, Morioka T, et al Development of hairy cell leukemia in familial platelet disorder with predisposition to acute myeloid leukemia. Platelets. 2014;25(4):300-302

23. Nickels EM, Soodalter J, Churpek JE, Godley LA. Recognizing familial myeloid leukemia in adults. Ther Adv Hematol. 2013;4(4):254 269

24. Latger-Cannard V, Philippe C, Bouquet A, et al. Haematological spectrum and genotypephenotype correlations in nine unrelated families with RUNX1 mutations from the French network on inherited platelet disorders. Orphanet J Rare Dis. 2016;11:49.

25. Challen GA, Goodell MA. Runx1 isoforms show differential expression patterns during hematopoietic development but have similar functional effects in adult hematopoietic stem cells. Exp Hematol. 2010;38(5):403-416.

26. Komeno Y, Yan M, Matsuura S, et al. Runx exon 6-related alternative splicing isoforms differentially regulate hematopoiesis in mice. Blood. 2014;123(24):3760-3769.

27. Brady G, Elgueta Karstegl C, Farrell PJ. Novel function of the unique $\mathrm{N}$-terminal region of RUNX1c in B cell growth regulation. Nucleic Acids Res. 2013;41(3):1555-1568

28. Lacaud G, Gore L, Kennedy M, et al. Runx1 is essential for hematopoietic commitment at the hemangioblast stage of development in vitro. Blood. 2002;100(2):458-466.

29. Navarro-Montero O, Ayllon V, Lamolda M, et al. RUNX1c regulates hematopoietic differentiation of human pluripotent stem cells possibly in cooperation with proinflammatory signaling. Stem Cells. 2017;35(11):22532266.

30. Lorsbach RB, Moore J, Ang SO, et al. Role of RUNX1 in adult hematopoiesis: analysis of RUNX1-IRES-GFP knock-in mice reveals differential lineage expression. Blood. 2004;103(7):2522-2529.

31. Tsuzuki S, Hong D, Gupta R, et al. Isoformspecific potentiation of stem and progenito cell engraftment by AML1/RUNX1. PLoS Med. 2007;4(5):e172

32. Tsuzuki S, Seto M. Expansion of functionally defined mouse hematopoietic stem and progenitor cells by a short isoform of RUNX1/AML1. Blood. 2012;119(3):727-735

33. Elagib KE, Racke FK, Mogass $M$, et al RUNX1 and GATA-1 coexpression and cooperation in megakaryocytic differentiaion. Blood. 2003:101(11):4333-4341.

34. Kuvardina ON, Herglotz J, Kolodziej S, et al. RUNX1 represses the erythroid gene expres- sion program during megakaryocytic differentiation. Blood. 2015;125(23):3570-3579.

35. Li Y, Jin C, Bai H, et al. Human NOTCH4 is a key target of RUNX1 in megakaryocytic differentiation. Blood. 2018;131(2):191-201.

36. Bellissimo DC, Speck NA. RUNX1 Mutations in inherited and sporadic leukemia. Front Cell Dev Biol. 2017:5:111

37. Gaidzik VI, Bullinger L, Schlenk RF, et al RUNX1 mutations in acute myeloid leukemia: results from a comprehensive genetic and clinical analysis from the AMI study group. J Clin Oncol. 2011;29(10):13641372

38. Tang JL, Hou HA, Chen CY, et al AML1/RUNX1 mutations in 470 adult patients with de novo acute myeloid leukemia: prognostic implication and interaction with other gene alterations. Blood. 2009;114(26:5352-5361

39. Bean LJH, Hegde MR. Clinical implications and considerations for evaluation of in silico algorithms for use with ACMG/AMP clinical variant interpretation guidelines. Genome Med. 2017:9(1):111.

40. Ghosh R, Oak N, Plon SE. Evaluation of in silico algorithms for use with ACMG/AMP clinical variant interpretation guidelines. Genome Biol. 2017:18(1):225.

41. Ioannidis NM, Rothstein JH, Pejaver V, et al REVEL: an ensemble method for predicting the pathogenicity of rare missense variants. Am J Hum Genet. 2016;99(4):877-885

42. Zhao LJ, Wang YY, Li G, et al. Functional features of RUNX1 mutants in acute transformation of chronic myeloid leukemia and their contribution to inducing murine fullblown leukemia. Blood. 2012;119(12):28732882

43. Tsai SC, Shih LY, Liang ST, et al. Biological activities of RUNX1 mutants predict secondary acute leukemia transformation from chronic myelomonocytic leukemia and myelodysplastic syndromes. Clin Cancer Res. 2015;21(15):3541-3551

44. Osato M, Asou N, Abdalla E, et al. Biallelic and heterozygous point mutations in the runt domain of the AML1/PEBP2alphaB gene associated with myeloblastic leukemias. Blood. 1999;93(6):1817-1824

45. Taketani T, Taki T, Takita J, et al. Mutation of the AML1/RUNX1 gene in a transient myeloproliferative disorder patient with Down syndrome. Leukemia. 2002;16(9): 1866-1867.

46. Koh CP, Wang CO, Ng CE, et al. RUNX1 meets MLL: epigenetic regulation of hematopoiesis by two leukemia genes. Leukemia. 2013;27(9):1793-1802.

47. Mersch J, Brown N, Pirzadeh-Miller S, et al. Prevalence of variant reclassification following hereditary cancer genetic testing. JAMA. 2018;320(12):1266-1274

48. Slavin TP, Manjarrez S, Pritchard CC, Gray $\mathrm{S}$, Weitzel JN. The effects of genomic germline variant reclassification on clinical cancer care. Oncotarget. 2019;10(4):417-423,

49. Michaud J, Wu F, Osato M, et al. In vitro analyses of known and novel RUNX1/AML1 mutations in dominant familial platelet disorder with predisposition to acute myelogenous leukemia: implications for mechanisms of pathogenesis. Blood. 2002:99(4):1364-1372.

50. Yeo G, Burge CB. Maximum entropy modeling of short sequence motifs with applications to RNA splicing signals. J Comput Biol. 2004;11(2-3):377-394.

51. Desmet FO, Hamroun D, Lalande M, et al. Human Splicing Finder: an online bioinformatics tool to predict splicing signals. Nucleic Acids Res. 2009;37(9):e67. 
52. Cavalcante de Andrade Silva M, Krepischi ACV, Kulikowski LD, et al. Deletion of RUNX1 exons 1 and 2 associated with familial platelet disorder with propensity to acute myeloid leukemia. Cancer Genet. 2018;222-223:32-37.

53. Papaemmanuil E, Gerstung M, Bullinger L, et al. Genomic classification and prognosis in acute myeloid leukemia. N Engl J Med. 2016;374(23):2209-2221.

54. Luddy RE, Champion LA, Schwartz AD. A fatal myeloproliferative syndrome in a family with thrombocytopenia and platelet dysfunction. Cancer. 1978;41(5):1959-1963.

55. Dowton SB, Beardsley D, Jamison D, Blattner S, Li FP. Studies of a familial platelet disorder. Blood. 1985;65(3):557-563.

56. Liew E, Owen C. Familial myelodysplastic syndromes: a review of the literature. Haematologica. 2011;96(10):1536-1542.

57. Miyoshi H, Shimizu K, Kozu T, et al. t( $8 ; 21)$ breakpoints on chromosome 21 in acute myeloid leukemia are clustered within a limited region of a single gene, AML1. Proc Natl Acad Sci U S A. 1991;88(23):10431-10434.

58. Erickson P, Gao J, Chang KS, et al. Identification of breakpoints in $\mathrm{t}(8 ; 21)$ acute myelogenous leukemia and isolation of a fusion transcript, AML1/ETO, with similarity to Drosophila segmentation gene, runt. Blood. 1992;80(7):1825-1831.

59. Feurstein S, Drazer MW, Godley LA. Genetic predisposition to leukemia and other hematologic malignancies. Semin Oncol. 2016;43(5):598-608.

60. Churpek JE, LA G. How I diagnose and manage individuals at risk for inherited myeloid malignancies. Blood. 2016;128 (14):1800-1813.

61. Shlush LI, Zandi S, Mitchell A, et al. Identification of pre-leukaemic haematopoietic stem cells in acute leukaemia. Nature. 2014;506(7488):328-333.

62. Arends CM, Galan-Sousa J, Hoyer K, et al. Hematopoietic lineage distribution and evolutionary dynamics of clonal hematopoiesis. Leukemia. 2018;32(9):1908-1919.
63. Thol F, Klesse S, Kohler L, et al. Acute myeloid leukemia derived from lymphomyeloid clonal hematopoiesis. Leukemia. 2017;31(6):1286-1295.

64. Petti AA, Williams SR, Miller CA, et al. Mutation detection in thousand of acute myeloid leukemia cells using single cell RNA-sequencing. bioRxiv. 2018 October 17. [Epub ahead of Print]

65. Velten L, Story BA, Hernancez-Malmierca P et al. MutaSeq reveals the transcriptomic consequences of clonal evolution in acute myeloid leukemia. bioRxiv. 2018 December 21 [Epub ahead of Print]

66. Wehr C, Grotius K, Casadei S, et al. A novel disease-causing synonymous exonic mutation in GATA2 affecting RNA splicing. Blood. 2018;132(11):1211-1215

67. Hsu AP, Johnson KD, Falcone EL, et al. GATA2 haploinsufficiency caused by mutations in a conserved intronic element leads to MonoMAC syndrome. Blood. 2013;121(19):3830-38371

68. Pippucci T, Savoia A, Perrotta S, et al Mutations in the 5' UTR of ANKRD26, the ankirin repeat domain 26 gene, cause an autosomal-dominant form of inherited thrombocytopenia, THC2. Am J Hum Genet. 2011;88(1):115-120.

69. Noris P, Perrotta S, Seri M, et al. Mutations in ANKRD26 are responsible for a frequent form of inherited thrombocytopenia: analysis of 78 patients from 21 families. Blood. 2011;117(24):6673-6680.

70. Azzariti DR, Riggs ER, Niehaus A, et al. Points to consider for sharing variant-leve information from clinical genetic testing with ClinVar. Cold Spring Harb Mol Case Stud. 2018;4(1)

71. Riggs ER, Azzariti DR, Niehaus A, et al Development of a consent resource for genomic data sharing in the clinical setting. Genet Med. 2019;21(1):81-88

72. Ma N, Zhang JZ, Itzhaki I, et al Determining the pathogenicity of a genomic variant of uncertain significance using CRISPR/Cas9 and human-induced pluripo- tent stem cells. Circulation. 2018;138(23): 2666-2681.

73. Sander JD, Joung JK. CRISPR-Cas system for editing, regulating and targeting genomes. Nat Biotechnol. 2014;32(4):347 355.

74. Tsai SQ, Joung JK. Defining and improving the genome-wide specificities of CRISPRCas9 nucleases. Nat Rev Genet 2016;17(5):300-312

75. Zhang L, Li Z, Yan J, et al. Mutagenesis of the Runt domain defines two energetic hot spots for heterodimerization with the core binding factor beta subunit. J Biol Chem. 2003;278(35):33097-33104.

76. Li Z, Yan J, Matheny CJ, et al. Energetic contribution of residues in the Runx1 Runt domain to DNA binding. J Biol Chem. 2003;278(35):33088-33096.

77. Findlay GM, Boyle EA, Hause RJ, Klein JC, Shendure J. Saturation editing of genomic regions by multiplex homology-directed repair. Nature. 2014;513(7516):120-123.

78. Findlay GM, Daza RM, Martin B, et al Accurate classification of BRCA1 variants with saturation genome editing. Nature. 2018;562(7726):217-222

79. Matreyek KA, Starita LM, Stephany JJ, et al Multiplex assessment of protein variant abundance by massively parallel sequencing. Nat Genet. 2018;50(6):874-882

80. Mester JL, Ghosh R, Pesaran T, et al. Genespecific criteria for PTEN variant curation: recommendations from the ClinGen PTEN Expert Panel. Hum Mutat. 2018;39(11):1581 1592.

81. Garrett LT, Hickman N, Jacobson A, et al Family studies for classification of variants of uncertain classification: current laboratory clinical practice and a new web-based educational tool. J Genet Couns. 2016;25(6): 1146-1156.

82. Bravo J, Li Z, Speck NA, Warren AJ. The leukemia-associated AML1 (Runx1)--CBF beta complex functions as a DNA-induced molecular clamp. Nat Struct Biol. 2001;8 (4):371-378. 